ARTICLE

Received 22 Apr 2016 | Accepted 11 Oct 2016 | Published 24 Nov 2016

DOI: $10.1038 /$ ncomms13517

OPEN

\title{
The Robo4 cytoplasmic domain is dispensable for vascular permeability and neovascularization
}

Feng Zhang ${ }^{1, \star}$, Claudia Prahst $^{1, \star}$, Thomas Mathivet ${ }^{2}$, Laurence Pibouin-Fragner ${ }^{2}$, Jiasheng Zhang $^{1}$, Gael Genet ${ }^{1}$, Raymond Tong ${ }^{3}$, Alexandre Dubrac ${ }^{1} \&$ Anne Eichmann $1,2,4$

Vascular permeability and neovascularization are implicated in many diseases including retinopathies and diabetic wound healing. Robo4 is an endothelial-specific transmembrane receptor that stabilizes the vasculature, as shown in Robo4 ${ }^{-/-}$mice that develop hyperpermeability, but how Robo4 signals remained unclear. Here we show that Robo4 deletion enhances permeability and revascularization in oxygen-induced retinopathy (OIR) and accelerates cutaneous wound healing. To determine Robo4 signalling pathways, we generated transgenic mice expressing a truncated Robo4 lacking the cytoplasmic domain (Robo4 $\Delta C D$ ). Robo4 $\triangle C D$ expression is sufficient to prevent permeability, and inhibits OIR revascularization and wound healing in Robo4 ${ }^{-/}$mice. Mechanistically, Robo4 does not affect Slit2 signalling, but Robo4 and Robo4 $\triangle C D$ counteract Vegfr2-Y949 (Y951 in human VEGFR2) phosphorylation by signalling through the endothelial UNC5B receptor. We conclude that Robo4 inhibits angiogenesis and vessel permeability independently of its cytoplasmic domain, while activating VEGFR2-Y951 via ROBO4 inhibition might accelerate tissue revascularization in retinopathy of prematurity and in diabetic patients.

\footnotetext{
${ }^{1}$ Cardiovascular Research Center, Yale University School of Medicine, Department of Internal Medecine Cardiology, New Haven, Connecticut 06510-3221, USA. ${ }^{2}$ INSERM U970, Paris Center for Cardiovascular Research (PARCC), 75015 Paris, France. ${ }^{3}$ Genentech Inc., Molecular Oncology Division, South San Francisco, California 94030, USA. ${ }^{4}$ Department of Cellular and Molecular Physiology, Yale University School of Medicine, New Haven, Connecticut 06510-3221, USA. * These authors contributed equally to this work. Correspondence and requests for materials should be addressed to A.E. (email: anne.eichmann@yale.edu).
} 
R OBO4 was identified 15 years ago in a bioinformatics search for endothelial-specific transcripts and initially named Magic Roundabout, to denote its homology to Robo receptors and its selective expression on endothelial cells in actively growing tumour vessels ${ }^{1}$. Robo 4 encodes a $140 \mathrm{kDa}$ protein that comprises a $60 \mathrm{kDa}$ extracellular domain (ECD) with two immunoglobulin (Ig)-like domains and two fibronectin-repeat regions, a transmembrane domain and an intracellular domain (ICD) devoid of known catalytic sequences ${ }^{2}$. Robo4 is upregulated in endothelium of embryonic blood vessels and in micro-vessels of patients with tumours and vascular injury, but is also seen in quiescent endothelium $^{1-7}$. Its endothelial-specific expression is regulated by activator protein-1 (AP-1) binding to a $3 \mathrm{~kb}$ fragment of the promoter ${ }^{7-9}$. Besides endothelial cells, Robo4 is also expressed in hematopoietic stem cells and regulates their trafficking ${ }^{10-13}$. The highly endothelial-specific Robo4 expression has prompted considerable interest in this molecule to selectively target or image tumour vascularization ${ }^{9,14-16}$.

Robo4 ${ }^{-1-}$ mice are viable and fertile, demonstrating that Robo4 function is dispensable for developmental angiogenesis ${ }^{17,18}$. Adult Robo4 ${ }^{-/-}$mice exhibit enhanced corneal neovascularization induced by VEGF implantation in the eyes, and Robo4 knockout breast tissue implanted into pregnant mice develops more vessels when compared with wildtype controls ${ }^{18,19}$, suggesting that Robo4 has anti-angiogenic effects in certain tissue contexts. In addition, Robo4 also maintains vascular barrier function, as shown in Robo4 ${ }^{-/-}$ mice, which have increased VEGF-induced dermal permeability $^{17,18}$. Furthermore Robo4 ${ }^{-1-}$ mice show increased angiogenesis in oxygen-induced retinopathy (OIR) ${ }^{17}$. OIR is widely used to model ocular neovascular disease ${ }^{20,21}$. Neonatal mice are subjected to $75 \%$ oxygen exposure for 5 days (P7-P12), resulting in vascular obliteration. After being placed back to room air, OIR retinas enter into a proliferative phase, leading to regrowth of normal vessels (revascularization) and formation of pathological pre-retinal neovascular tufts driven by angiogenic factors. Thus, OIR models can be used to examine vessel loss, physiological revascularization and pathological neovascular tuft formation $^{20,21}$.

VEGF signals permeability induction by activating a specific tyrosine residue in the VEGFR2 intracellular domain, Y951 (Y949 in mouse Vegfr2). The pY951 residue mediates binding of the T cell-specific adapter (TSAd), which is essential for VEGF-induced c-Src activation, vascular permeability and pathological angiogenesis $^{22-25}$. Mutant mice carrying a substitution of Y949 to phenylalanine, and TSAd knockout mice are viable and fertile, but fail to show VEGF-induced Src phosphorylation and are deficient in VEGF- but not histamine-induced permeability in vivo ${ }^{23-25}$. They also show normal developmental angiogenesis, but decreased tumour vascularization ${ }^{23-25}$.

How Robo4 signals to maintain vascular barrier function is currently unclear. Initial studies reported that Robo4 acts as a receptor for Slit family proteins (Slit1-3) that regulate crossover of axons at the mid-line of the developing central nervous system $^{2,17,26-29}$ and also mediate cardiovascular development and angiogenesis ${ }^{15,30-33}$. Slit2 binding to Robo4 was proposed to counteract VEGF-driven angiogenesis and vascular permeability by signalling through the Robo $4 \mathrm{ICD}^{17}$ that can bind to paxillin and other cytoskeletal modifying proteins ${ }^{2,34,35}$. However, Slit2 does not bind to Robo4 in Biacore binding assays, and Slit2 binding to vasculature is unaltered in $R o b o 4^{-/-}$mice $^{14,18}$. In fact, crystal structure analysis showed that the critical Slit-binding residues in the Robo1 and 2 extracellular domains are not conserved in mammalian Robo3 and 4 (refs 36-39). Alternatively, Robo4 could affect angiogenesis by modulating Slit2 signalling through Robol and 2. A recent study showed that tamoxifen-inducible deletion of Slit2 in postnatal mice leads to severe retinal angiogenesis defects, demonstrating potent proangiogenic functions for Slit2 (ref. 32). Tamoxifen-inducible deletion of Robo2 on a Robo1-null background phenocopies the Slit2 mutant defects, demonstrating that Slit2 provides proangiogenic signals via Robol and 2 (ref. 32). These in vivo studies support pro-angiogenic Slit effects previously observed in vitro ${ }^{40-45}$. ROBO1 and 4 can be co-immunoprecipitated from HUVEC lysates, suggesting that Robo4 could restrain angiogenesis by acting as a dominant negative regulator of Slit2 signalling through Robol and 2 (ref. 35).

A third model of Robo4 action is that it signals through UNC5B, another endothelial-specific guidance receptor ${ }^{46-49}$. High-throughput screening of $>1,500$ secreted proteins and ECD domains with a Robo4-ECD-domain fused to the Fc portion of human Immunoglobulin (Robo4-ECD-Fc) identified the UNC5B-ECD-Fc as the only Robo4-binding protein ${ }^{18}$. The Robo4 ECD binds to the UNC5B ECD with a Kd of $12 \mathrm{nM}$ (ref. 18). Robo4-ECD-Fc binds to cells transfected with UNC5B, and Robo4 binding to blood vessels is lost in $U n c 5 B^{-/-}$mice, together providing strong evidence for a direct interaction between both receptors. The Robo4 ECD activates signalling through Unc5B, which inhibits Src activation downstream of Vegfr2, thereby attenuating VEGF-mediated sprouting angiogenesis $^{18}$. These data suggest that the Robo4 ECD might be sufficient to mediate Robo4 actions in the vasculature.

To distinguish between the different possible modes of Robo4 action, we generated mice lacking the Robo4 cytoplasmic signalling domain. If this domain was endowed with signalling capacity, mice should develop a phenotype resembling Robo4 knockouts. However, we find that the Robo4 cytoplasmic domain is dispensable for its effect on angiogenesis. We show here that Robo4 ${ }^{-1-}$ mice have increased ocular permeability and revascularization when subjected to OIR and exhibit accelerated healing of cutaneous wounds. Robo $4 \Delta \mathrm{CD}$ expression inhibits OIR revascularization, vessel permeability and wound healing in Robo4 knockout mice. Signalling studies show that the ROBO4 ECD signals via the UNC5B ICD to prevent activation of VEGFR2 Y951. The data suggest that promoting Y951 activation via $\mathrm{ROBO} 4$ blockade might represent an opportunity to enhance ocular revascularization in retinopathy of prematurity and wound healing in diabetic patients.

\section{Results}

Generation of mice lacking the Robo4 cytoplasmic domain. We used a mouse Robo $4 \Delta C D$ cDNA construct (aa 1-522) that expressed the ECD and TM domain but lacked $>95 \%$ of ICD sequence and was fused to GFP (Supplementary Fig. 1). The construct was placed under the control of a tetracyclineresponsive element (TET-off system) and used to generate transgenic mice. These mice were crossed with a CDH5promoter-driven tetracycline-transactivator (CDH5-tTA) transgenic mouse line ${ }^{50}$. The double transgenic mice (hereafter Robo ${ }^{+/+} ;$Robo4ACD mice) were expected to express CDH5driven Robo4 $\triangle \mathrm{CD}$ in endothelial cells and additionally expressed endogenous Robo4. Mice carrying either of the single transgenes expressed endogenous Robo4 but not Robo4 $\triangle C D$ and were used as controls (Robo4 ${ }^{+/+}$;Stg mice) (Fig. 1a). We also generated mice expressing Robo $4 \Delta \mathrm{CD}$ in the absence of endogenous Robo4 (hereafter Robo4 ${ }^{-/-} ;$Robo4 $\triangle C D$ mice), by intercrossing the double transgenic mice with Robo4 $4^{-/-}$mice ${ }^{18}$.

Robo4 ${ }^{+/+} ;$Robo4 $4 C D$ and Robo4 ${ }^{-/-} ;$Robo4 $4 C D$ mice were born at the expected Mendelian frequency in the absence of doxycycline treatment (Fig. 1b), indicating that neither lack of the full-length protein, nor lack of its cytoplasmic domain affected embryonic vascular development. Western blot analysis with an 
a

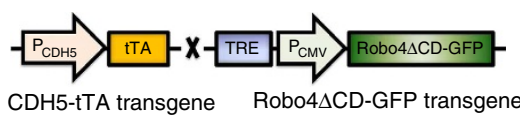

C

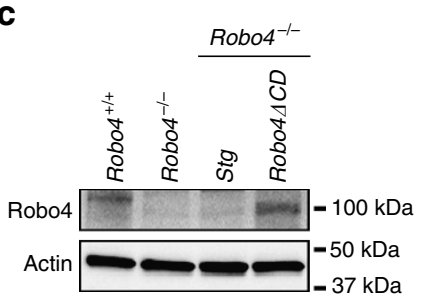

\begin{tabular}{lccc}
\hline Genotype & $\begin{array}{c}\text { No } \\
\text { transgene }\end{array}$ & $\begin{array}{c}\text { CDH5-tTA or Robo4 } 4 \text { CD } \\
\text { single transgenic }\end{array}$ & $\begin{array}{c}\text { Double } \\
\text { transgenic }\end{array}$ \\
\hline $\begin{array}{l}\text { Robo4 } \\
\text { Observed/expected }\end{array}$ & $82 / 92.5$ & $189 / 185$ & $99 / 92.5$ \\
$\begin{array}{l}\text { Robo4 } \\
\text { Observed/expected }\end{array}$ & $46 / 51.5$ & $107 / 103$ & $53 / 51.5$ \\
\hline
\end{tabular}

d

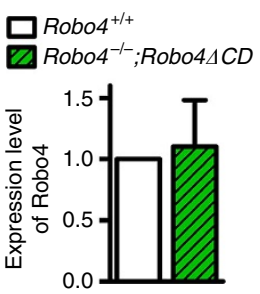

e

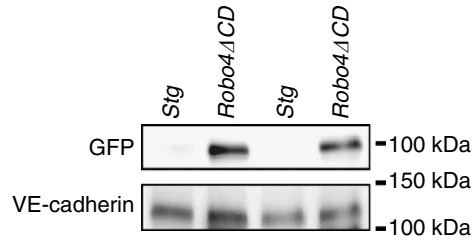

f
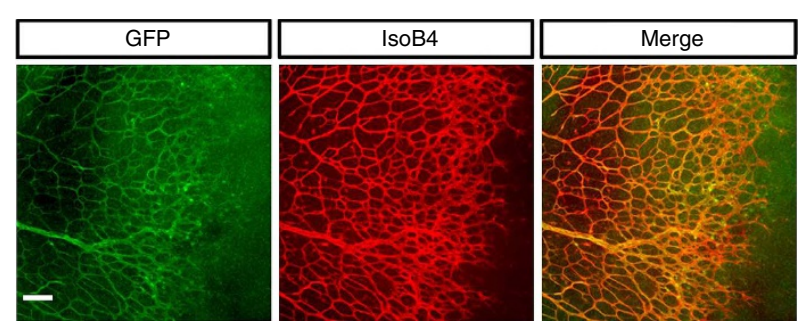

g
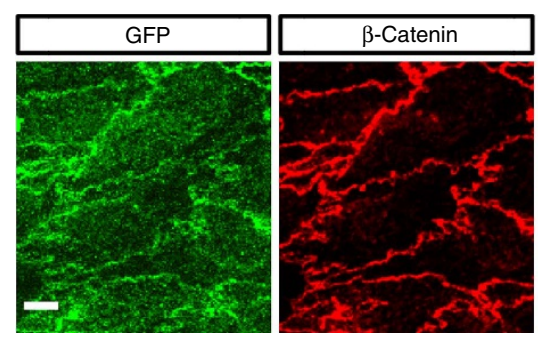

j

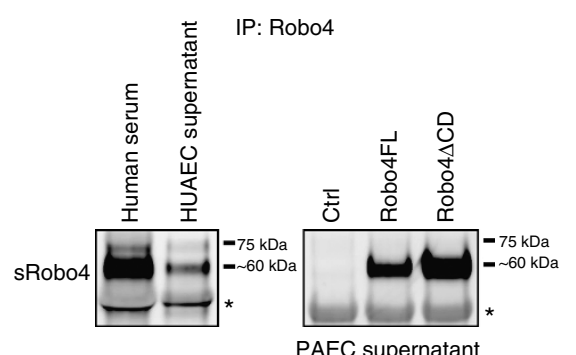

PAEC supernatant

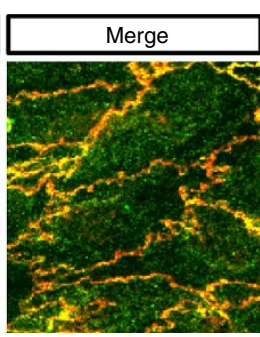

Mouse serum k

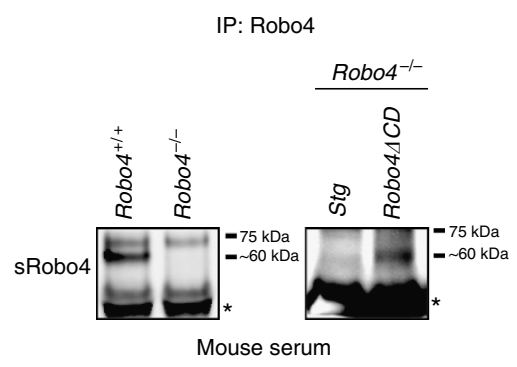

h

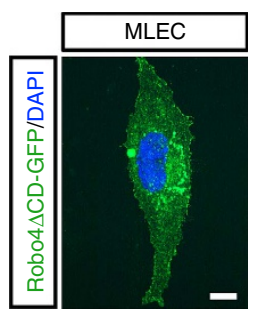

i

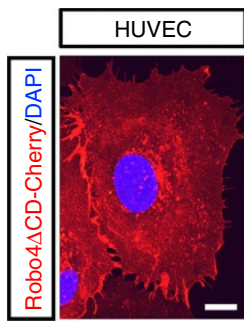

$\mathbf{\nabla}$ Robo4 $^{+/+}$

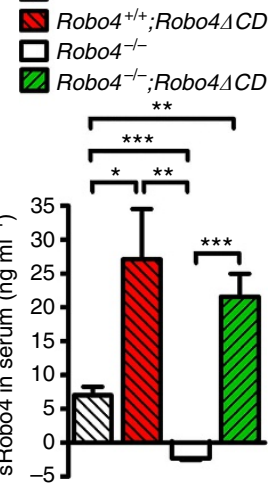

Figure 1 | Generation and characterization of Robo4 $\Delta$ CD transgenic mice. (a) Schematic of transgenic constructs. (b) Mendelian distribution of transgenic mice. 370 mice (53 litters) on Robo4 ${ }^{+/+}$background and 206 mice (31 litters) on Robo4 $4^{-/}$background were genotyped. (c) Anti-Robo4 western blot on MLECs isolated from the indicated mice. Note lower molecular weight ( $100 \mathrm{kDa})$ of Robo4 $\Delta$ CD-GFP than endogenous Robo4 ( $\sim 140 \mathrm{kDa}$ ). (d) Quantification of blots in c. $N=3$ mice per group. (e) Western blot with anti-GFP on mouse lung lysates. Each lane shows one lung lysate of the indicated genotype (all on Robo4 ${ }^{+/+}$background). (f) Vascular-specific anti-GFP staining of a P7 Robo4 ${ }^{+} /+; R o b 04 \Delta C D$ retina counterstained with IsoB4. Scale bar, $100 \mu \mathrm{m}$. (g) En face anti-GFP staining of aortic endothelium of an adult Robo4 ${ }^{+/+}$;Robo4 $\Delta C D$ mouse counterstained with $\beta$-catenin. Scale bar, $20 \mu \mathrm{m}$. (h) Membrane GFP staining in Robo4 ${ }^{+/+} ;$Robo4 $\triangle C D$ MLEC. (i) Membrane and junctional labelling of HUVECs infected with Robo4 $\Delta C D$-mCherry adenovirus. Scale bar, $10 \mu \mathrm{m}$. (j) Anti-Robo4 IP and western blot with human serum and cell culture supernatants from HUAECs and PAEC transfected with the indicated constructs. Note presence of a soluble Robo4 (sRobo4) band at $\sim 60 \mathrm{kDa}$. Lower bands ( ${ }^{*}$ ) correspond to serum Ig. (k) SRobo4 is absent in Robo4 ${ }^{-/-}$and Robo4 ${ }^{-/-}$;Stg mouse serum but detected in Robo4 ${ }^{+/+}$and Robo4 ${ }^{-/-}$;Robo4ACD mouse serum. (I) sRobo4 expression analysis by ELISA with mouse serum from 6-8 week old mice. $N=10-17$ mice in each group. The data represent mean \pm s.e.m. ${ }^{\star} P<0.05$; ${ }^{\star \star} P<0.01 ;{ }^{* \star \star} P<0.001$, Welch's $t$-test. 
anti-Robo4 antibody on mouse lung endothelial cell (MLEC) protein extracts confirmed the presence of a single $140 \mathrm{kDa}$ Robo4 band in wild-type mice, lack of expression in Robo4 ${ }^{-/-}$ and Robo4 ${ }^{-/-}$;Stg mice and expression of a shorter $\sim 100 \mathrm{kDa}$ Robo $4 \Delta \mathrm{CD}$ form corresponding to the ECD $(60 \mathrm{kDa}), \mathrm{TM}$ domain and GFP (together approximately $40 \mathrm{kDa}$ ) in cells isolated from Robo4 ${ }^{-/}$;Robo4 $\triangle C D$ mice (Fig. 1c). Quantification of western blots showed that expression levels of Robo4 $\Delta C D$ were similar to endogenous Robo4, indicating that transgenic mice expressed physiological levels of the mutant protein (Fig. 1d). Because it was GFP-tagged, the $100 \mathrm{kDa}$ Robo $4 \Delta \mathrm{CD}$ protein could also be detected by western blot with an anti-GFP antibody in mouse lung lysates from Robo4 ${ }^{+/+} ;$Robo4 $4 C D$ mice but not from Robo4 ${ }^{+{ }^{+}}$;Stg mice (Fig. 1e). Anti-GFP staining of Robo4 ${ }^{+/+}$;Robo4 $4 C D$ postnatal retina vasculature, adult aorta endothelium and primary MLECs showed specific transgene expression in endothelial cells (Fig. 1f-h). The Robo4 $\Delta$ CD-GFP fusion protein was primarily localized on the cell surface, with enrichment in the lateral junctional areas (Fig. 1g,h). Expression of mCherry-tagged Robo4 $\triangle \mathrm{CD}$ construct in HUVECs confirmed cell surface localization of the mutant protein (Fig. 1i; Supplementary Fig. 2).

We also detected a soluble Robo4 (sRobo4) form of $60 \mathrm{kDa}$ using immunoprecipitation with an anti-Robo4 antibody in human serum and in supernatant collected from human umbilical artery ECs (HUAECs, Fig. 1j). Based on its molecular weight, sRobo4 is likely to encode most of the ECD. Expression of Robo4-full length (FL) and $\triangle \mathrm{CD}$ constructs in porcine aortic endothelial cells (PAECs) led to sRobo4 secretion into the supernatant, whereas untransfected PAECs did not express sRobo4 (Fig. 1j). sRobo4 was also detected in mouse serum from wild-type and Robo4 ${ }^{-/-} ;$Robo4 $4 C D$ mice, but was absent in Robo4 ${ }^{-/-}$mice (Fig. 1k). ELISA analysis of serum confirmed the presence of sRobo4 in Robo4 ${ }^{+/+} ;$Robo $4 \Delta C D$ mice but not in Robo4 $4^{-/-}$mice (Fig. 1l). The serum level of sRobo4 is $\sim 7 \mathrm{ng} \mathrm{ml}^{-1}$ in Robo4 ${ }^{+/+}$mice, $\sim 25 \mathrm{ng} \mathrm{ml}^{-1}$ in Robo4 ${ }^{+/+} ;$Robo4 $4 C D$ and $\sim 20 \mathrm{ng} \mathrm{ml}^{-1}$ in Robo4 ${ }^{-/-} ;$Robo4 $4 C D$ mice, demonstrating moderate overexpression of sRobo4 in transgenic mice (Fig. 1l).

Robo4 $\Delta C D$ inhibits OIR revascularization. To assess effects of Robo $4 \Delta C D$ on developmental angiogenesis, we analysed postnatal retinas of Stg and Robo4 $\Delta C D$ expressing mice on both wild-type and Robo4 knockout background. We did not see any differences in vessel outgrowth and vessel branching between Robo4 $\Delta C D$ expressing mice and their control littermates at P7 (Supplementary Fig. 3a).

We next challenged mice with OIR. After hyperoxia exposure, P12 Robo4 ${ }^{+/+}, R_{0 b} 4^{-/-}, R_{0 b} 4^{-/-}$;Stg and Robo4 ${ }^{-/-}$; Robo4 $\triangle C D$ pups all developed comparable vaso-obliteration, leading to a capillary-free avascular area in the centre of the retina (Supplementary Fig. 3b,c). Hence, Robo4 did not affect hyperoxia-induced vaso-obliteration.

After return to room air, hypoxia in the avascular area triggered re-growth of normal vessel sprouts from centrally located veins and the remaining capillaries in the periphery (Fig. 2a,b), and pre-retinal neovascular tufts (Fig. 2e, see arrowheads for tufts). Compared with Robo4 ${ }^{+/+}$, Robo4 ${ }^{-1-}$ mice showed increased revascularization, characterized by a significant decrease of the retina avascular area, and increased sprouting from veins (Fig. 2a-d). Expression of Robo $4 \Delta C D$ in Robo4 ${ }^{-9_{-}}$mice prevented the increased revascularization (Fig. 2a-d), indicating that Robo4 $\Delta$ CD rescued Robo4 function during ocular revascularization. Intraperitoneal injection of recombinant sRobo4 protein encoding His-tagged $\mathrm{ECD}^{18}$ in Robo4 ${ }^{-/}$mice inhibited OIR revascularization (Fig. 2a-d), demonstrating that sRobo4 is sufficient to activate signalling.
Interestingly, neovascular tuft formation was unchanged in Robo4 ${ }^{-I^{-}}$and Robo4 ${ }^{-/-} ;$Robo4 $4 C D$ mice and after sRobo4 injection (Fig. 2e,f), indicating that Robo4 controlled revascularization but was dispensable for pathological neovascular tuft formation in the OIR model.

To examine vascular leak, we injected P17 OIR Robo4 ${ }^{+/+}$, Robo4 $4^{-/-} ;$Stg and Robo4 ${ }^{-/-} ;$Robo4 $4 C D$ mice retro-orbitally with $70 \mathrm{kDa}$ rhodamine-dextran and fluorescent Alexa 647 conjugated IsoB4, which labelled the luminal endothelial membrane of perfused vessels. Dyes were left to circulate for $5 \mathrm{~min}$, then retinas were harvested and re-stained with Alexa 488 conjugated IsoB4 (Fig. 3a,b). Overlay of injected and stained IsoB4 revealed efficient perfusion in all genotypes (Fig. 3a-c). Robo4 ${ }^{-/-}$;Stg mice showed increased vessel leak, as attested by reduced dextran labelling of Alexa 647 IsoB4 + retinal vasculature compared with wild-type mice (Fig. 3b,d). Dextran labelling of Robo4 $4^{-/-} ;$Robo4 $\triangle C D$ retinas was similar to that seen in wild-type mice, demonstrating that Robo4 $\triangle C D$ was sufficient to rescue vascular leak (Fig. 3b,d).

Robo4 $\Delta C D$ inhibits vascular leak and wound healing. As Robo4 ${ }^{-/}-$mice exhibit enhanced VEGF-driven dermal vessel permeability $^{17,18}$, we tested effects of Robo $4 \Delta C D$ in a Miles assay. To this end, Evans blue was injected intravenously followed by an intradermal injection of saline or VEGF into Robo4 ${ }^{+/+}$, Robo4 ${ }^{-1-} ;$ Stg and Robo4 ${ }^{-1-} ;$ Robo4 $4 C D$ mice. After $30 \mathrm{~min}$, animals were sacrificed, perfused with PBS and extravasated dye was imaged and quantified. With saline injection, Robo4 ${ }^{-/-}$;Stg mice exhibited slightly increased leakage of the Evans blue dye into the skin when compared with Robo4 ${ }^{+/+}$and Robo $4^{-/-}$;Robo4ACD mice (Fig. 4a,b). Injection of VEGF into the control wild-type mice led to a robust leakage of Evans Blue (Fig. 4a,b). Robo4 ${ }^{-1-}$;Stg exhibited significantly increased leakage of the Evans blue dye into the skin (Fig. 4a,b). In contrast, VEGF injection caused much less dye leakage in Robo4 $4^{-/-}$;Robo4 $\triangle C D$ mice (Fig. 4a,b). To test whether this effect was specific for the VEGF pathway, we injected another potent inducer of vessel permeability, histamine. Histamineinduced permeability was similar in $R_{0 b o 4}{ }^{+/+} ;$Robo $4 \Delta C D$ and in Robo4 ${ }^{+l+} ;$ Stg mice (Supplementary Fig. 4), suggesting that Robo $4 \Delta C D$ specifically targets VEGF-induced permeability signalling and is sufficient to prevent this process.

To determine the functional outcome of enhanced revascularization and vessel permeability in Robo4 ${ }^{-/}$mice, we next studied cutaneous wound healing. Robo4 $4^{-/-}$mice and Robo4 ${ }^{-1-} ;$ Stg mice exhibited significantly faster wound closure than wild-type littermates at 7 days after wounding (Fig. 4c-e). Expression of Robo4 $\Delta \mathrm{CD}$ in Robo4-null background was sufficient to prevent accelerated wound healing (Fig. 4c-e).

ROBO4 $\triangle$ CD decreases VEGFR2 Y951 phosphorylation. To address ROBO4 effects on VEGF signalling, we knocked down ROBO4 in HUVECs with siRNA (Supplementary Table 1), which strongly decreased $\mathrm{ROBO} 4$ protein levels (Fig. 5a). ROBO4 silencing accelerated VEGF-induced HUVEC monolayer permeability (Supplementary Fig. 5a), wound closure (Supplementary Fig. 5b,c) and increased vascularization in three-dimensional (3D) fibrin gels (Supplementary Fig. 5d,e).

To determine the mechanism responsible for the enhanced VEGF response, we treated control and ROBO4 siRNA knockdown cells with VEGF for 5 and $15 \mathrm{~min}$ and examined phosphorylation of the VEGFR2 intracellular tyrosine residues Y951, Y1175 and Y1214. VEGF induced a significant increase of phosphorylation at all sites in control siRNA treated cells (Fig. 5a; 
Supplementary Fig. 6a). ROBO4 knockdown further enhanced phosphorylation on Y951, but not on Y1175 or Y1214 (Fig. 5a-c; Supplementary Fig. 6a). Phosphorylated VEGFR2 Y951 activates
c-Src ${ }^{23,24}$, and c-Src phosphorylation levels were also increased in ROBO4 knockdown cells (Fig. 5a,d). Likewise, VEGF treatment of primary MLECs showed enhanced activation of Vegfr2 pY949

a

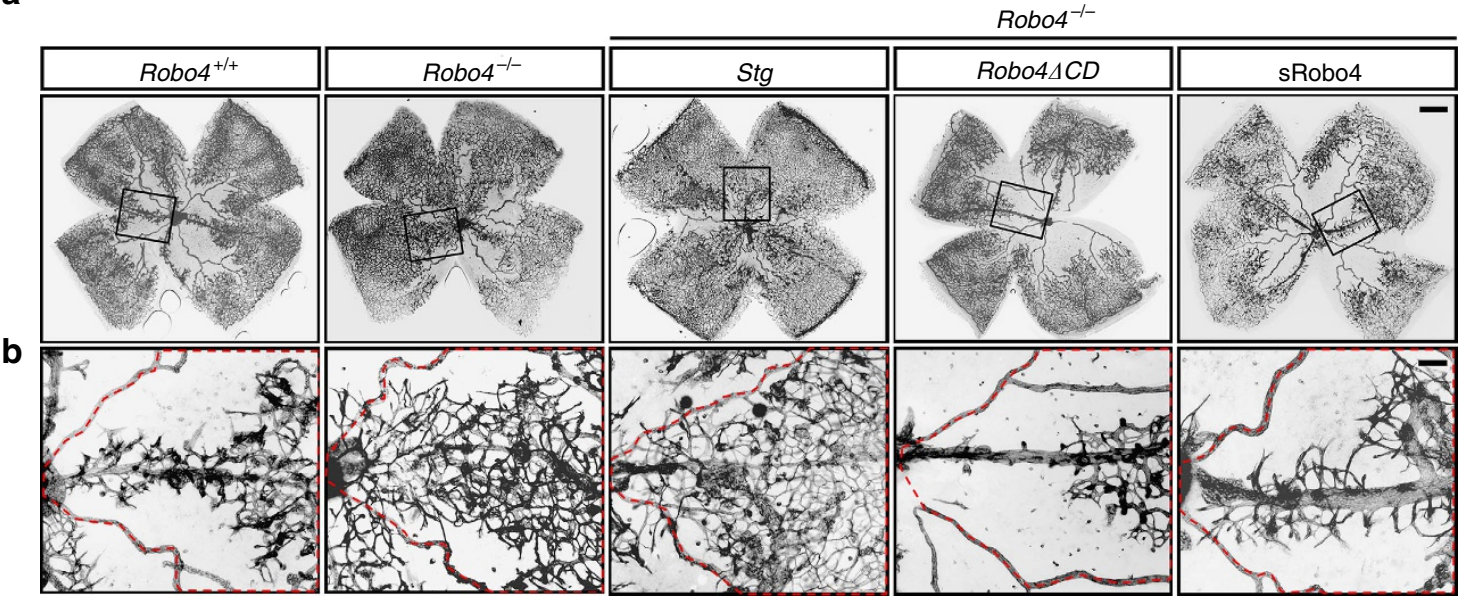

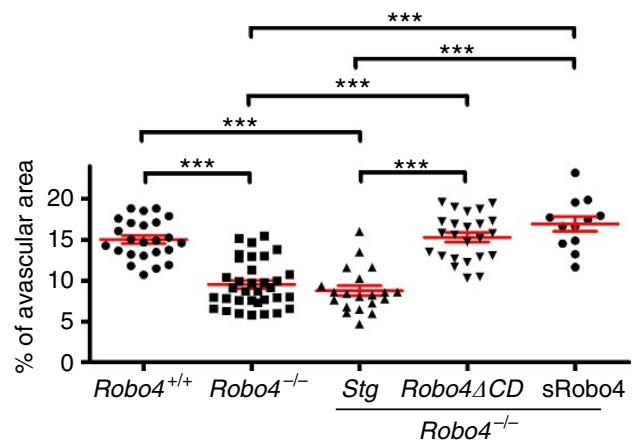

d

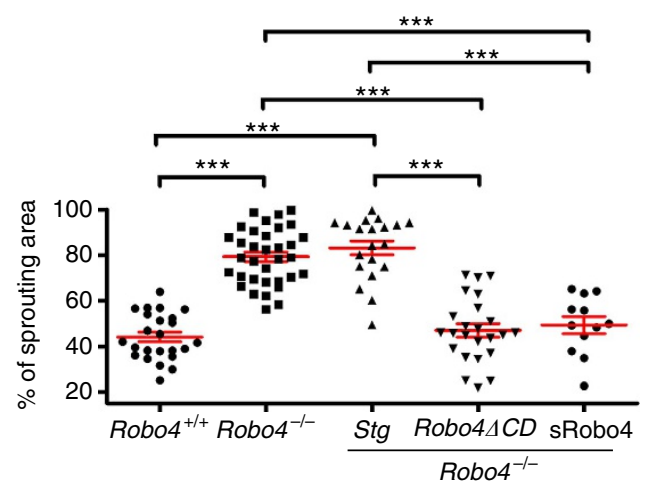

e

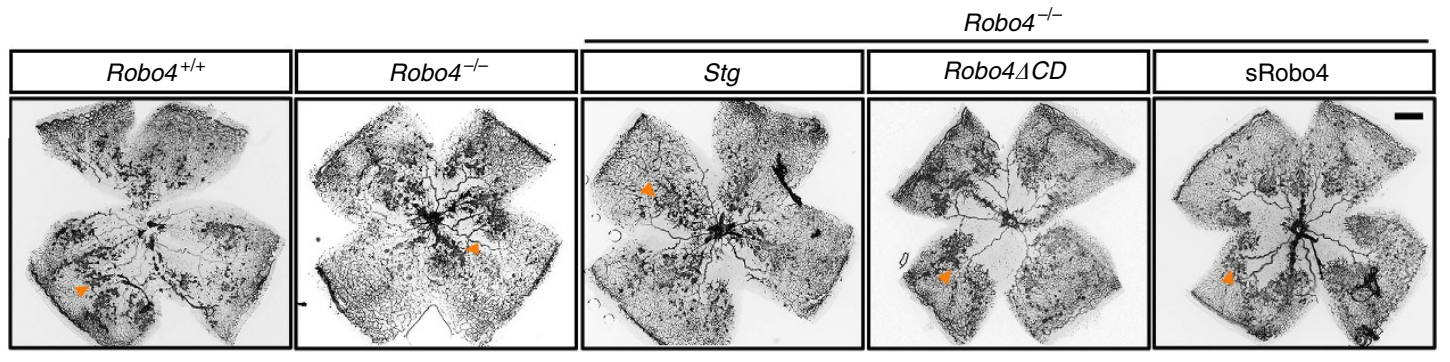

f

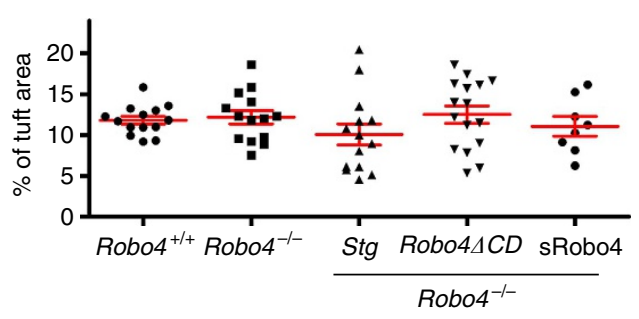

Figure 2 | Robo4 inhibits OIR revascularization independently of its cytoplasmic domain. (a) IsoB4-stained whole-mount retinal vasculature of P17 mice with the indicated genotypes after OIR. SRobo4 was injected intraperitoneally daily from P12 to P16. Scale bar, $500 \mu$ m. (b) Higher magnification images of boxed areas in a showing vessels sprouting from veins. Scale bar, $100 \mu \mathrm{m}$. (c) Quantifications of avascular area in the whole retinas shown in a. (d) Quantifications of vessel coverage in the red stippled area in $\mathbf{b}$. Each dot represents a retina. $N=12-34$ retinas (6-17 mice) per group. Error bars: s.e.m. ${ }^{\star \star \star} P<0.001$, Mann-Whitney $U$ test. (e) IsoB4-stained neovessel tufts of P17 mice with the indicated genotypes after OIR. To preferentially stain neovascular tufts (arrowheads), retinas were subjected to mild detergent permeabilization and short IsoB4 incubation. Scale bar, $500 \mu \mathrm{m}$.

(f) Quantifications of tuft area in the retinas shown in e. $N=8-16$ retinas (4-8 mice) per group. Error bars: s.e.m. No significant differences in tuft area were observed using Mann-Whitney $U$ test. 
and Src phosphorylation in cells isolated from Robo4 ${ }^{-1-}$ mice compared with Robo4 ${ }^{+1+}$ mice, while Y1173 activation was similar between genotypes (Fig. 5e-h).

Treatment of ROBO4 knockdown HUVECs with recombinant sRobo4 was sufficient to reduce VEGF-induced Y951 activation, suggesting that the ROBO4 cytoplasmic domain was dispensable for effects on VEGF signalling (Supplementary Fig. 6b). To test this further, we re-expressed siRNA-resistant full-length mouse Robo4 in ROBO4 knockdown HUVECs, which prevented the increase in VEGF-induced Y951 phosphorylation (Fig. 5i,j; Supplementary Table 1). Next, we expressed siRNA-resistant mouse Robo4 $\triangle \mathrm{CD}$ in $\mathrm{ROBO} 4$ knockdown HUVECs, which was sufficient to prevent the excessive pY951 and p-Src in response to VEGF (Fig. 5k,l; Supplementary Table 1). Consistently, both constructs also inhibited increased HUVEC monolayer permeability, wound closure and fibrin gel sprouting angiogenesis

a

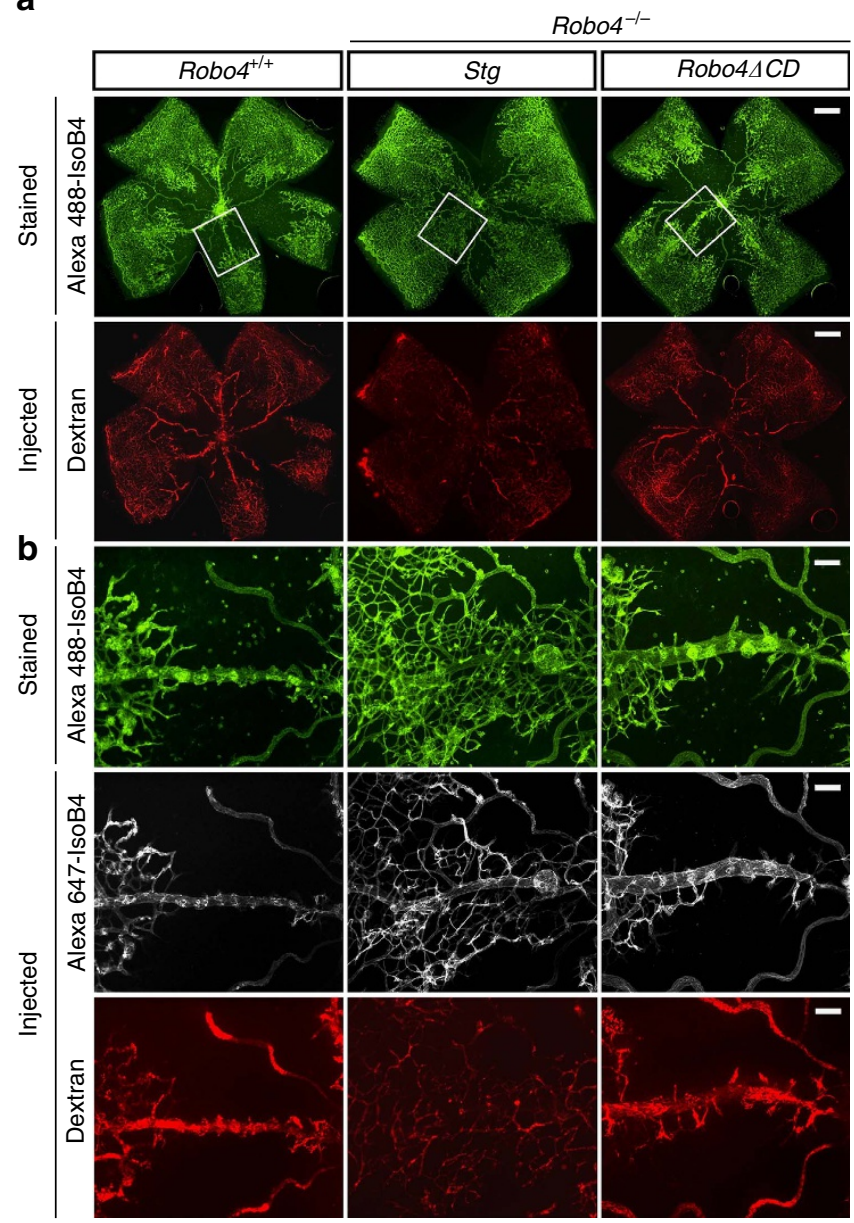

C

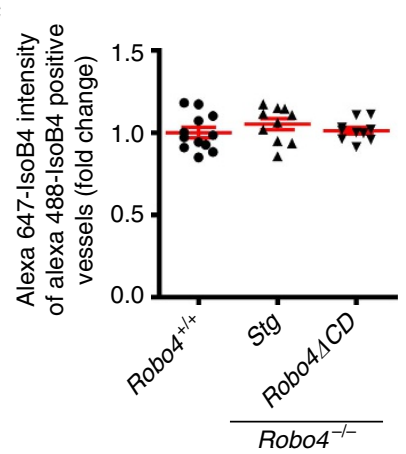

d

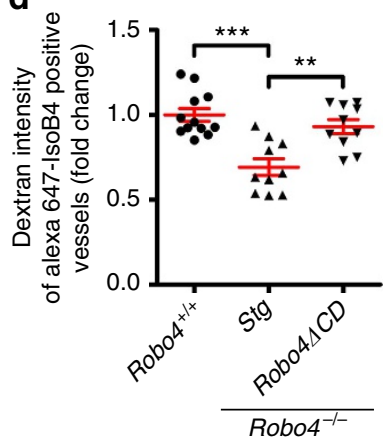

seen with VEGF treated, ROBO4-silenced cells (Supplementary Fig. 5). In primary MLECs, we observed that VEGF-induced Y949 and Src phosphorylation are reduced in $R o b o 4^{-/-}$; Robo4 $4 C D$ mice compared with Robo4 ${ }^{-/-}$;Stg littermates, while Y1173 activation is similar between genotypes (Fig. 5m,n; Supplementary Fig. 6c). Thus, ROBO4 selectively targets the VEGFR2 Y951/949-Src signalling cascade independently of its cytoplasmic domain (Fig. 5o).

ROBO4 is dispensable for Slit2 signalling. To determine if enhanced angiogenesis in the absence of Robo 4 could be due to dominant negative effects on Slit2 signalling through Robo1, we tested if ROBO4 affected Slit2 signalling in HUVECs in vitro (Fig. 6a). ROBO4 siRNA knockdown in HUVECs did not affect mRNA expression levels of ROBO1 and 2 (Supplementary Fig. 7). Stimulation with recombinant Slit2 and western blot analysis showed that Slit2 induced AKT phosphorylation, and that $\mathrm{ROBO} 4$ silencing did not affect the level of Slit2-induced p-AKT (Fig. 6b). Expression of Robo4 $\triangle \mathrm{CD}$ in $\mathrm{ROBO} 4$ siRNA knockdown cells did not affect Slit2-induced AKT activation (Fig. 6c). We next tested effects of ROBO4 on Slit2-induced angiogenic sprouting in 3D fibrin gels. Again, neither ROBO4 siRNA, nor Robo $4 \triangle \mathrm{CD}$ affected sprouting induced by Slit2 (Fig. 6d). In contrast to ROBO4, silencing of $R O B O 1$ and 2 abolished Slit2-induced AKT activation (Fig. 6e). Reconstitution of $R O B O 1$ and 2 knockdown cells with adenovirus encoding full-length siRNA-resistant rat Robo1 rescued Slit2-induced AKT activation (Fig. 6e; Supplementary Table 1). AKT activation of ROBO1 and 2 knockdown cells was not rescued by expression of Robo $\Delta \mathrm{CD}$ (Fig. 6e), indicating that the Robol ICD is required for Slit2 signalling while ROBO4 does not interfere with Slit2 mediated AKT activation and sprouting.

ROBO4 affects VEGFR2 pY951 via UNC5B. As ROBO4 binds to UNC5B and activates UNC5B signalling ${ }^{18}$, we next examined the effect of UNC5B on VEGF-induced pY951 (Fig. 7a). We knocked down UNC5B in HUVECs with siRNA (Supplementary Table 1), which strongly decreased UNC5B mRNA levels (Supplementary Fig. 7b). We found that UNC5B silencing also enhanced VEGF-induced VEGFR2 pY951 without affecting phosphorylation of the Y1175 residue (Fig. 7b,c). Re-expression

Figure 3 | Robo4 $\Delta C D$ inhibits OIR retinal vessel leakage. P17 OIR mice of the indicated genotypes were injected with Rhodamine-dextran and Alexa 647-IsoB4 for $5 \mathrm{~min}$, then mice were sacrificed and vasculature was stained with Alexa 488-IsoB4. (a) Low-magnification images of the Alexa 488-IsoB4-stained vasculature (upper panel, green) and Rhodamin-dextran perfusion (red, lower panel). Note reduced intensity of dextran labelling indicating increased permeability in Robo4 ${ }^{-/-}$;Stg mice compared with Robo4 ${ }^{+/+}$and Robo4 ${ }^{-/-} ;$Robo4 $4 C D$ mice. Scale bars, $500 \mu \mathrm{m}$. (b) Higher magnification images of the boxed areas in $\mathbf{a}$. Upper panel: Alexa 488-IsoB4-stained vasculature. Middle panel: injected Alexa 647-IsoB4-labelled vasculature. Lower panel: injected rhodamine-dextran labelled vasculature. Scale bars, $100 \mu \mathrm{m}$. (c) Quantifications of injected Alexa 647-IsoB4 fluorescence intensity in Alexa 488-IsoB4-labelled vasculature in images shown in $\mathbf{b}$. Each dot represents a retina. $N=10-12$ retinas (5-6 mice) per group. Error bars represent s.e.m. Note no significant difference between genotypes, indicating equal perfusion of all groups. (d) Quantification of dextran fluorescence intensity in Alexa 647-IsoB4labelled vasculature. Data were normalized to Alexa 647-IsoB4 intensity and are presented as fold change compared with Robo4 ${ }^{+/+}$mice. Robo4 ${ }^{-/-}$;Stg mice show reduced intensity of dextran labelling hence increased leakage, this is rescued in Robo4 ${ }^{-1-} ;$ Robo4 $\triangle C D$ mice. Each dot represents a retina. $N=10-12$ retinas (5-6 mice) per group. Error bars represent s.e.m. ${ }^{\star \star} P<0.01 ;{ }^{\star \star \star} P<0.001$, Mann-Whitney $U$ test. 
a

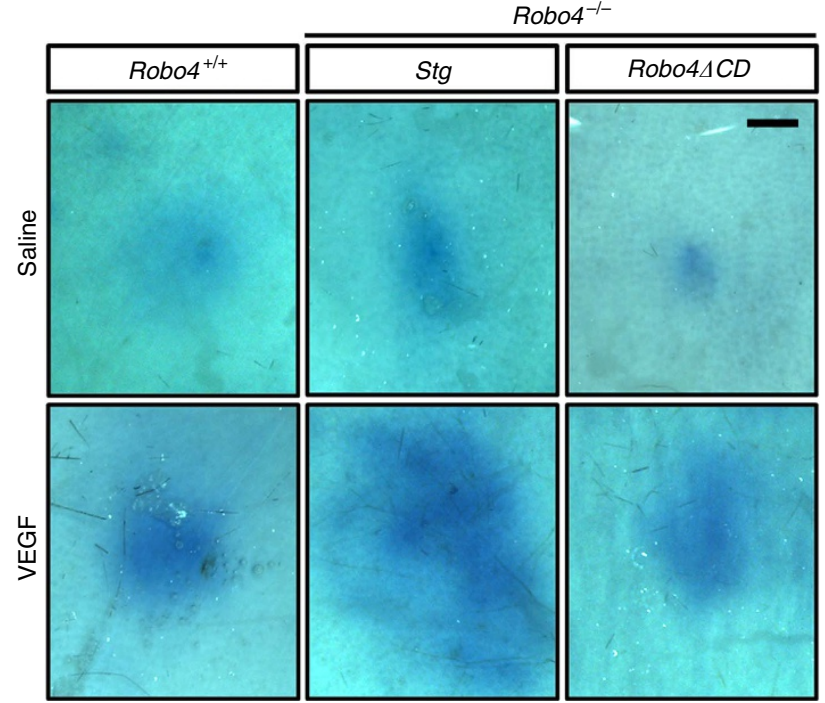

C

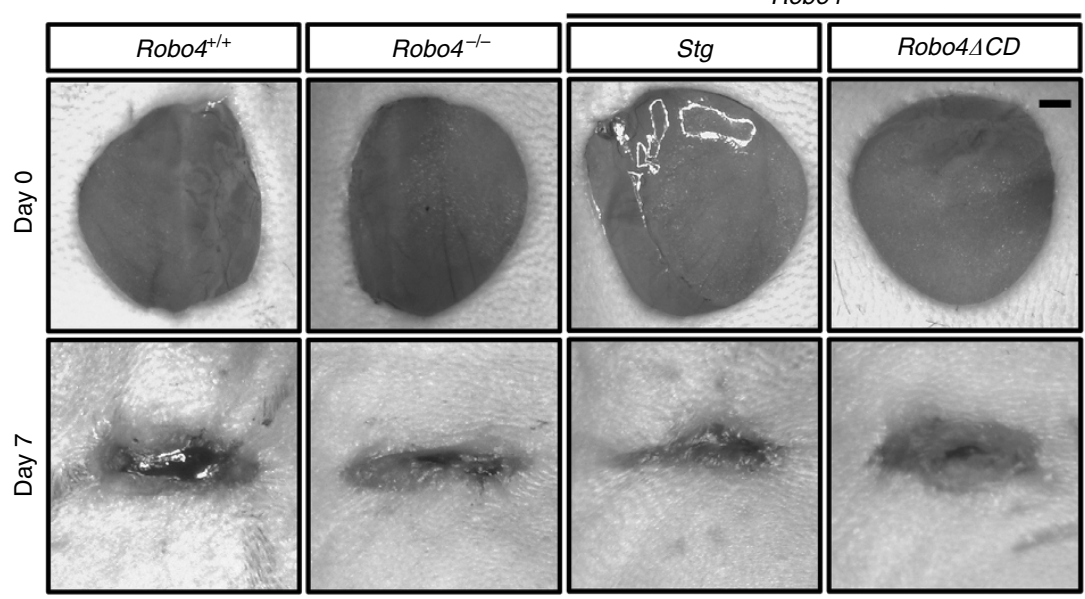

b

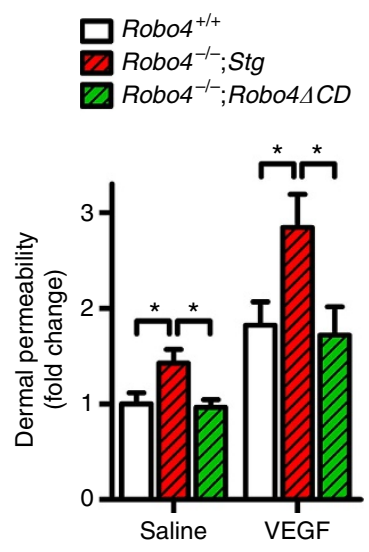

e

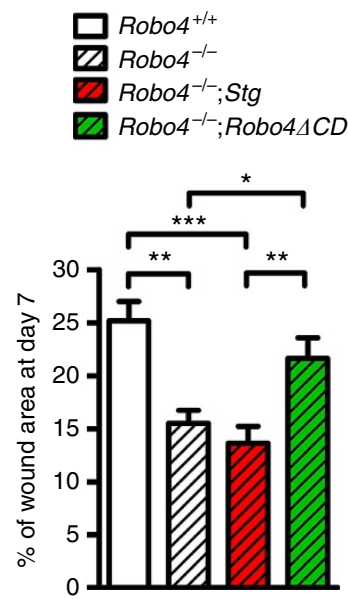

Figure 4 | Robo4 $\Delta C D$ inhibits dermal vascular permeability and wound healing. (a) Miles dermal permeability analysis. Evans blue leakage was assessed $30 \mathrm{~min}$ after intradermal injection of saline or $50 \mathrm{ng}$ VEGF into the indicated mice. Animals were PBS perfused before imaging and quantification. Note increased leak in VEGF treated Robo4 ${ }^{-/-}$;Stg compared with Robo4 ${ }^{+/+}$and Robo4 ${ }^{-/-} ;$Robo4ACD mice. Scale bar, 2 mm. (b) Quantification of Evans blue dye in the skin. $N=5-8$ mice per group. Error bars represent s.e.m. ${ }^{\star} P<0.05$, Mann-Whitney $U$ test. (c,d) Representative images of wound healing of mice with the indicated genotypes at day 0 and 7 after cutaneous punch biopsy. Scale bar, $1 \mathrm{~mm}$. (e) Quantification of percentage of wound area at day 7 after wounding. $N=14$ wounds (7 mice) in each group. Error bars: s.e.m. ${ }^{\star} P<0.05$; ${ }^{\star \star} P<0.01 ;{ }^{\star \star \star} P<0.001$, Mann-Whitney $U$ test.

of siRNA-resistant rat full-length Unc5B (rUnc5BFL, Fig. 7d; Supplementary Fig. 7c) in UNC5B-silenced cells rescued VEGFinduced VEGFR2 pY951 and Src phosphorylation (Fig. 7e,f). These data suggested that ROBO4 could affect VEGF signalling via UNC5B.

To understand which domains of UNC5B were required for VEGF signalling, we generated various Unc5B cytoplasmic domain deletions (Fig. 7d). Expression of a rat Unc5B construct deleted in its entire cytoplasmic domain ( $\mathrm{rUnc5B} \Delta \mathrm{CD}$ ) in UNC5B knockdown cells failed to rescue VEGF-induced VEGFR2 pY951 phosphorylation (Fig. 7g,h; Supplementary Fig. 7c), suggesting that the Unc5B signalling domain was required to modulate VEGFR2 activation. The Unc5B cytoplasmic domain contains a death domain (DD) implicated in cell survival, a UPA domain (abbreviated for conserved in Unc5B, Pidd and Ankyrin) and a ZU5 domain ${ }^{51}$ (Fig. 7d). Expression of rUnc5B $\Delta D D$ lacking the death domain in $U N C 5 B$ knockdown cells rescued VEGF-induced VEGFR2 pY951 phosphorylation (Fig. 7g,h; Supplementary Fig. 7c), indicating that the DD is dispensable for Unc5B effects on VEGFR2 signalling. In contrast, expression of rUnc5B $\triangle U P A$ in $U N C 5 B$ knockdown cells failed to rescue VEGF-induced VEGFR2 pY951 phosphorylation (Fig. 7g,h; Supplementary Fig. 7c), indicating that the UPA domain contains residues required for modulation of VEGFR2 signalling. To test if the UPA domain was sufficient to target VEGFR2 Y951, we generated a construct containing only the cytoplasmic rUnc5B UPA domain linked to Unc5B transmembrane and extracellular domain (Fig. 7d). Expression of this construct in UNC5B knockdown cells was sufficient to rescue VEGFR2 Y951 activation (Fig. 7g,h; Supplementary Fig. 7c). None of the Unc5B constructs affected VEGFR2 Y1175 phosphorylation, demonstrating a highly specific effect of the activated UPA domain on Y951 (Fig. 7g,h; Supplementary Fig. 7c). Finally, expression of rUnc5BFL in cells silenced for both $U N C 5 B$ and $R O B O 4$ failed to rescue Y951 activation (Fig. 7i,j), demonstrating that UNC5B cannot modulate VEGFR2 in the absence of ROBO4. For uncropped versions of all immunoblots shown in this study, see Supplementary Fig. 8. 
These data support a model in which ROBO4 decreases VEGFR2 Y951 activation by binding to UNC5B and activating signalling through the UNC5B UPA domain (Fig. 7a).

\section{Discussion}

We show here that Robo4 deletion enhances revascularization in OIR models and during cutaneous wound healing. Transgenic expression of near-physiological levels of Robo4 $\Delta \mathrm{CD}$ suppresses a
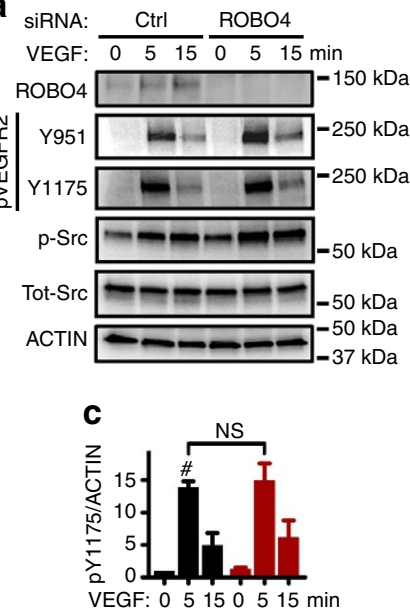

i

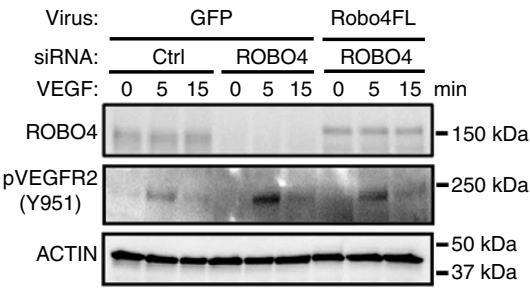

j Ctrl siRNA + adGFP

- ROBO4 siRNA + adGFP

- ROBO4 siRNA + adRobo4FL

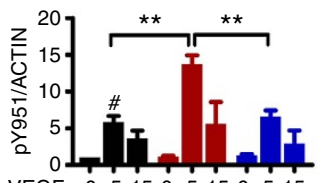

VEGF: $0551505150515 \mathrm{~min}$
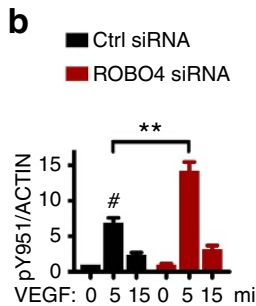

d

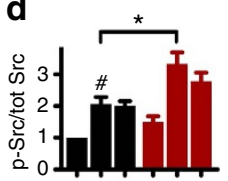

VEGF: $05150515 \mathrm{~min}$ e
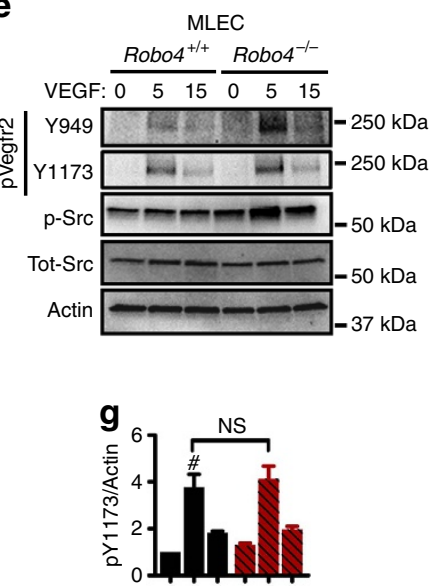

VEGF: $05150515 \mathrm{~min}$ f

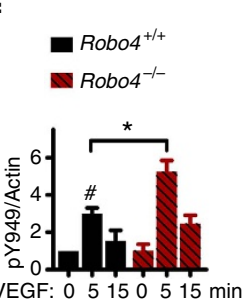

VEGF: $05150515 \mathrm{~min}$

k

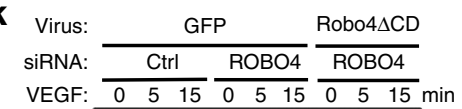

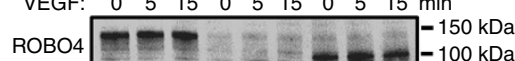
$\mathrm{ROBO4}$
$\mathrm{PVEGFR2}$
(Y951) p-Src $=-------50 \mathrm{kDa}$ Tot-Src $-5-50 \mathrm{kDa}$ $-50 \mathrm{kDa}$
$-50 \mathrm{kDa}$

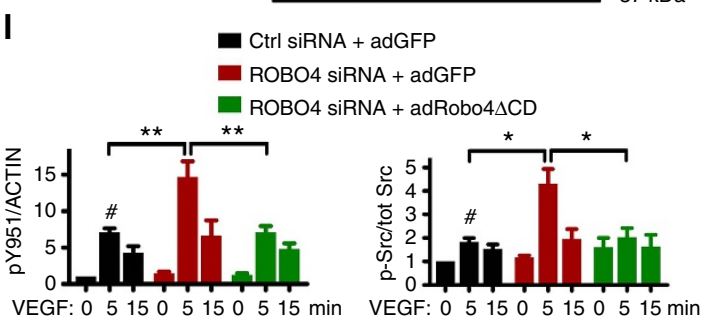

m

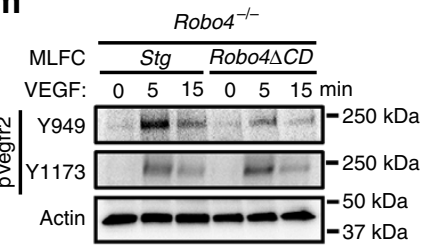

n

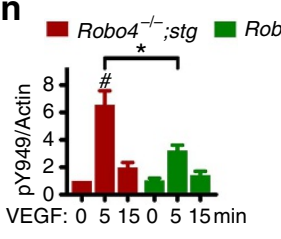

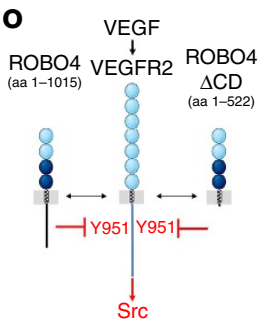

Figure 5 | Robo4 $\triangle$ CD affects VEGF signalling through VEGFR2 Y951. (a) Western blot analysis of VEGFR2 and Src phosphorylation in HUVECs transfected with Ctrl or ROBO4 siRNAs and treated with $3 \mathrm{nM}$ VEGF. (b-d) Quantifications of blots shown in a. \#: VEGF significantly induces VEGFR2 pY951, pY1175 and p-Src at 5 min. ROBO4 siRNA further increases pY951 and p-Src, but not pY1175. N=4-6 experiments. Error bars: s.e.m. NS, not significant; ${ }^{\star} P<0.05$; ${ }^{\star \star} P<0.01$, Student's $t$-test. (e) Western blot analysis of pVegfr2 and $\mathrm{p}$-Src in MLECs from Robo4 ${ }^{+/}+{ }^{+}$and Robo4 ${ }^{-/}-$mice after $6 \mathrm{nM}$ VEGF stimulation. (f-h) Quantifications of blots shown in e. \#: VEGF significantly induces Vegfr2 pY949 and pY1173. Robo4 ${ }^{-/-}$cells show further enhanced pY949 and p-Src, but not pY1173. $N=3$ experiments. Error bars: s.e.m. NS, not significant; ${ }^{\star} P<0.05$, Student's $t$-test. (i) Western blot and (j) quantification of pY951 in HUVECs with indicated siRNA transfection and adenovirus infection after $3 \mathrm{nM}$ VEGF stimulation. \#: VEGF significantly induces VEGFR2 pY951 at 5 min. ROBO4FL rescues increased pY951 in ROBO4 silenced cells. $N=4$ experiments. Error bars: s.e.m. ${ }^{\star \star} P<0.01$,

Student's t-test. (k) Western blot analysis of pY951 $(n=6)$ and p-Src $(n=4)$ and the corresponding quantifications (I) in HUVECs with indicated siRNA transfection and adenovirus infection after $3 \mathrm{nM}$ VEGF stimulation. \#: VEGF significantly induces VEGFR2 pY951 and p-Src at 5 min. Robo4 $\Delta C D$ restores pY951 and p-Src in ROBO4 silenced cells to Ctrl levels. Error bars: s.e.m. ${ }^{\star} P<0.05$, ${ }^{\star \star} P<0.01$, Student's $t$-test. (m) Western blot and (n) quantification of pVegfr2 in MLECs from Robo4 $4^{-/-} ;$Stg and Robo4 ${ }^{-/-} ;$Robo4 $4 C D$ mice after $6 \mathrm{nM}$ VEGF stimulation. Note decreased Y 949 but unchanged Y1173 in Robo4 ${ }^{-/-}$;Robo4 $\triangle C D . N=5$ experiments. Error bars: s.e.m. NS, not significant; ${ }^{\star} P<0.05$, Student's $t$-test. (o) Schematic of ROBO4 and Robo4 $\Delta C D$ effects on VEGF signalling through VEGFR2. 
a

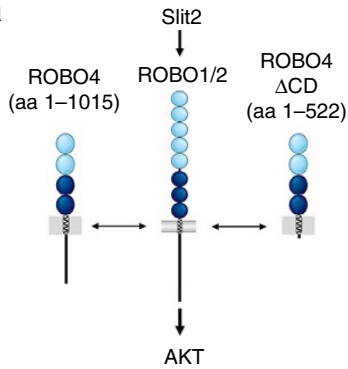

C

b

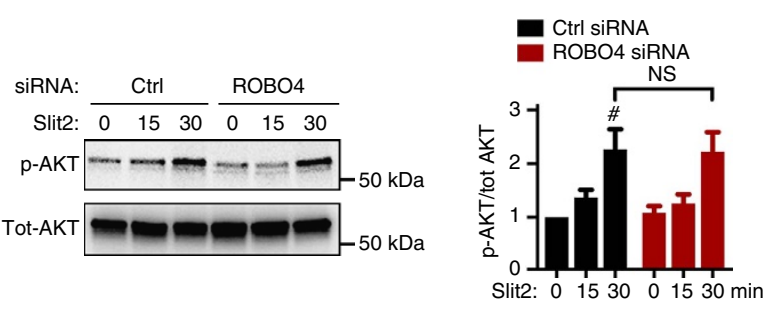

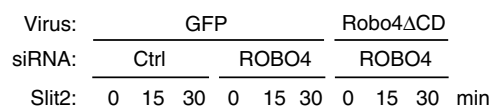

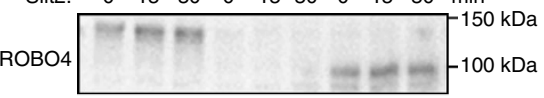

p-AKT $-\ldots-\ldots-\ldots$

Tot-AKT
Ctrl siRNA + adGFP

ROBO4 siRNA + adGFP

ROBO4 siRNA + adRobo4 $\triangle C D$

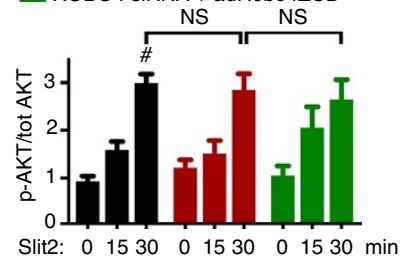

d GFP Robo4 $\triangle \mathrm{CD}$

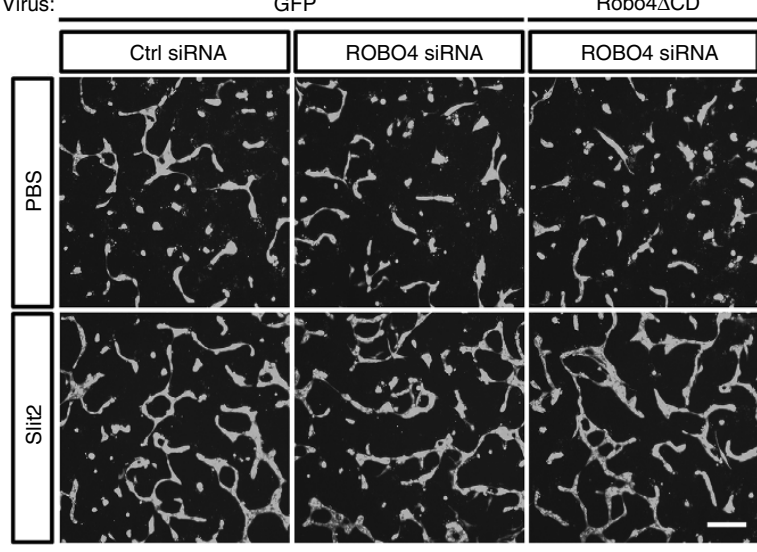

Ctrl siRNA + adGFP

ROBO4 siRNA + adGFP

ROBO4 siRNA + adRobo4 $\triangle C D$

$\left.0^{2.0}\right]$

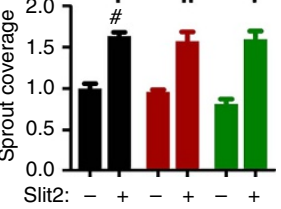

e

Ctrl siRNA + adGFP

- ROBO1\&2 siRNA + adGFP

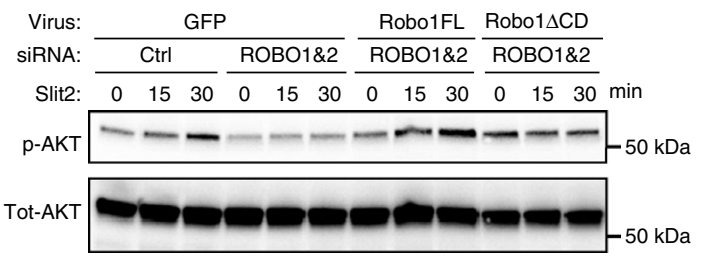

ROBO1\&2 siRNA + adRobo1 $\triangle C D$

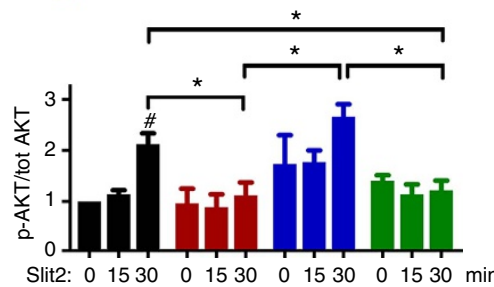

Figure 6 | ROBO4 $\triangle$ CD does not affect Slit2-mediated AKT activation and sprouting. (a) Schematic of possible ROBO4 and Robo4 $\triangle C D$ effects on Slit2-ROBO1/2 signalling. (b) Western blot of phospho-AKT S473 (p-AKT) (left) and quantification (right) in HUVECs with Ctrl or ROBO4 siRNA transfection and $6 \mathrm{nM}$ Slit2 stimulation. \#: Slit2 significantly induces $p-A K T$ at $30 \mathrm{~min} . \mathrm{N}=3$ experiments. Error bars: s.e.m. NS, not significant, Student's $t$-test. (c) Left panel: western blot analysis of ROBO4 expression and p-AKT in HUVECs with indicated siRNA transfection and adenovirus infection after $6 \mathrm{nM}$ Slit2 stimulation. Right panel: western blot quantifications of p-AKT/tot-AKT $(n=4)$. \#: Slit2 significantly induces p-AKT at 30 min. Error bars: s.e.m. NS, not significant, Student's t-test. (d) HUVEC sprouting in 3D fibrin gels (left) and the corresponding quantifications (right, $n=3$ ). Cells were treated with siRNAs and virus as indicated and then stimulated with $6 \mathrm{nM}$ Slit2 for $96 \mathrm{~h}$. \#: Slit2 significantly induces HUVEC sprouting. Error bars: s.e.m. NS, not significant, Mann-Whitney $U$ test. Scale bar, $200 \mu \mathrm{m}$. (e) Western blot analysis of p-AKT in HUVECs with indicated siRNA transfection and adenovirus infection after $6 \mathrm{nM}$ Slit2 stimulation. $N=3$ experiments. \#: Slit2 significantly induces $p$-AKT at 30 min. Error bars: s.e.m. ${ }^{\star} P<0.05$ when compared with Ctrl siRNA + adGFP group, Student's t-test.

VEGF-induced vessel permeability, excessive angiogenesis and would healing in Robo4 $4^{-1-}$ mutants. These results demonstrate that Robo4 restrains angiogenesis independently of its cytoplasmic domain.

The OIR retina undergoes two types of angiogenesis: revascularization and neovascular tuft formation ${ }^{20,21}$. Revascularization is considered as physiological 'healing' of the injured retina and leads to formation of normal vasculature that eliminates ischemia, while pathological neovascularization results in formation of fragile balloon-like vessels prone to bleeding, hence aggravating ischemic tissue injury ${ }^{20,21}$. Our results show that Robo4 blockade enhances OIR revascularization and vessel permeability without 
exacerbating pathological tuft formation. Thus, blocking ROBO4 might be beneficial to accelerate revascularization in ROP. Likewise, Robo4 blocking accelerates wound closure in endothelial monolayers and promotes cutaneous wound healing, suggesting that ROBO4 blockade could be used to promote wound healing in diabetic patients.
The data are inconsistent with a model where Robo4 signals through its ICD to suppress angiogenesis. Thus, even though the Robo4 ICD can interact with various cytoplasmic signalling molecules $2,26,34,35$, this association appears dispensable for the in vivo Robo4 effects described in this study. Immunolocalization studies in HUVECs had previously shown that full-length a

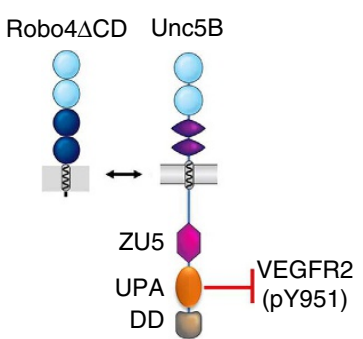

d

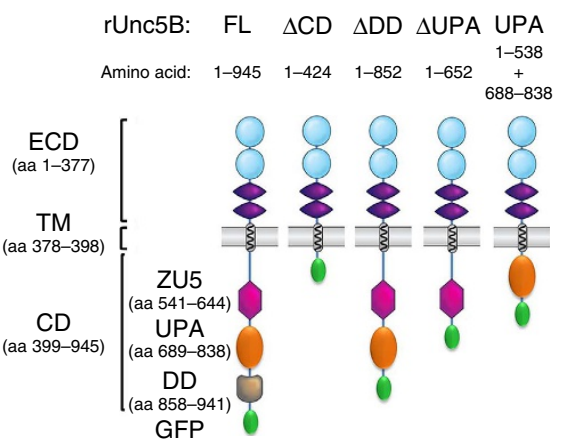

g

Virus:

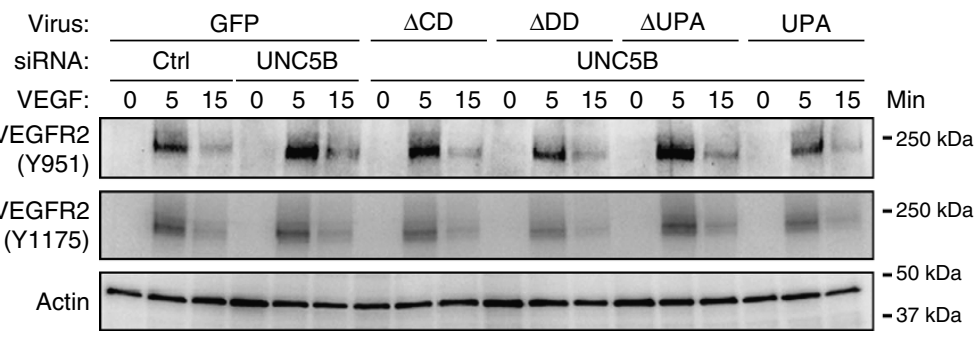

h

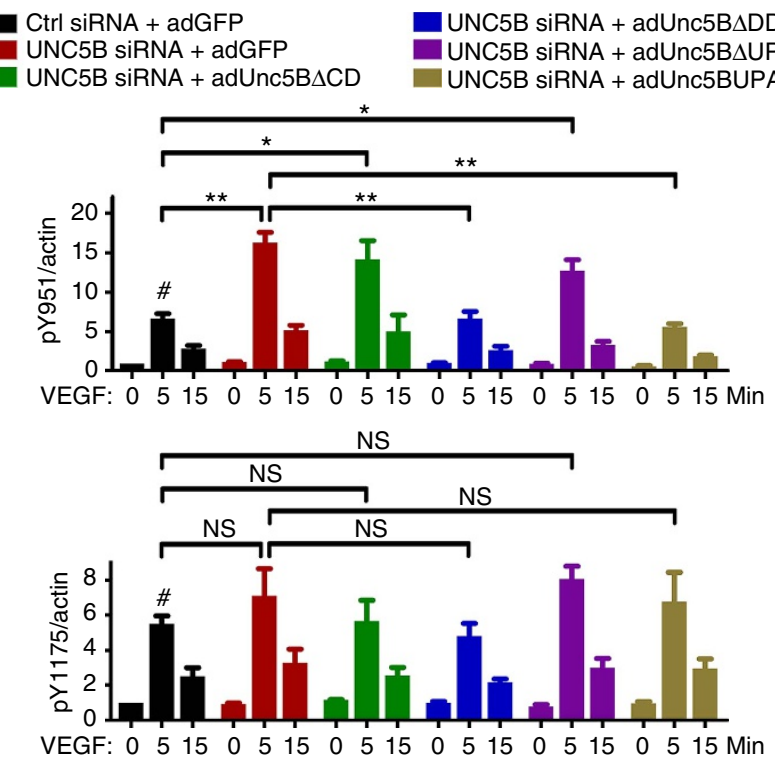

C

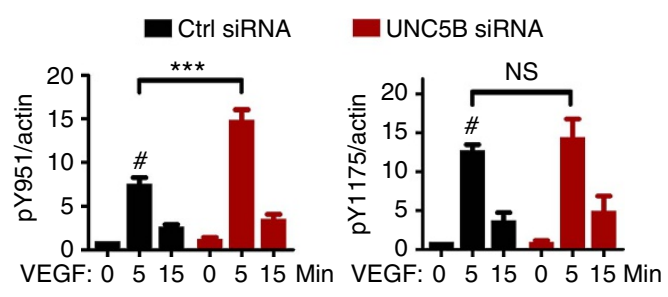

f

Ctrl siRNA + adGFP

UNC5B SiRNA + adGFP

UNC5B siRNA + adUnc5BFL

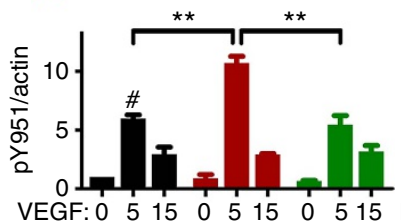

VEGF: 0515055150515 Min

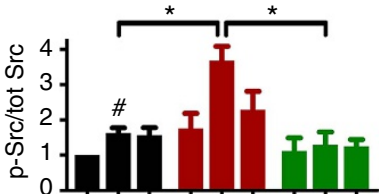

VEGF: $0 \begin{array}{llllllll}5 & 15 & 0 & 5 & 15 & 0 & 5 & 15\end{array}$ Min

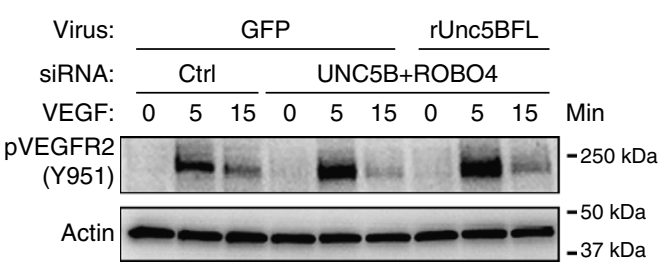

j

Ctrl siRNA + adGFP

ROBO4/UNC5B siRNA + adUnc5BFL

ROBO4/UNC5B siRNA + adGFP

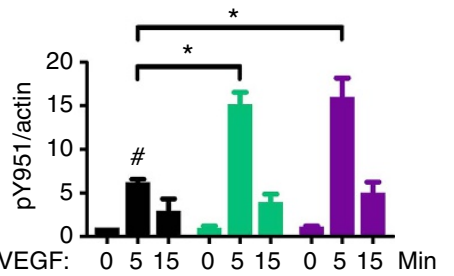


ROBO4 shuttles between the plasma membrane and intracellular vesicles, while Robo $4 \Delta \mathrm{CD}$ is readily detectable at the plasma membrane $e^{18,35}$. We speculate that the ROBO4 ICD may control its trafficking between intracellular vesicles and plasma membrane, perhaps via association with some of the previously identified binding partners, many of which are implicated in endocytosis and vesicle trafficking ${ }^{52-55}$.The molecular details of this remain to be elucidated, but appear of minor relevance for $\mathrm{ROBO} 4$ biological function.

Interestingly, we find that a soluble $60 \mathrm{kDa}$ form of Robo4 is detectable in supernatant from Robo4 expressing cells and in mouse and human serum. The size of sRobo4 suggests that it is a proteolytic fragment that contains most of the $\mathrm{ECD}$, including the $\mathrm{N}$-terminal Ig-like domains that bind UNC5B (see below) ${ }^{18}$. Thus, sRobo4 appears to be endowed with biological activity and its release into the circulation could activate Robo4 signalling to inhibit VEGF-induced permeability and angiogenesis. Increased plasma Robo4 levels in patients with tumours or vascular injury ${ }^{3,56}$ may therefore reflect a physiological strategy to protect vasculature from hyperpermeability and excessive angiogenesis. Alternatively, sRobo4 could interfere with the interaction between membrane-bound Robo4 and UNC5B and inhibit UNC5B signalling.

Having established that the ICD is dispensable for ROBO4 function, we tested if $\mathrm{ROBO} 4$ restrained angiogenesis by heterodimerization with ROBO1. MLECs and HUVECs express 10-fold higher levels of ROBO4 than of ROBO1 (ref. 32), and both receptors can be co-immunoprecipitated from endothelial cell lysates ${ }^{35}$, suggesting that ROBO4 could affect Slit2 signalling through ROBO1. Since ROBO4 lacks Slit2 binding residues ${ }^{36-39}$, its heterodimerization with ROBO1 could lead to formation of complexes unable to bind and transduce Slit2 signals. Inducible loss of Slit2 or combined deletion of Robo1 and 2 function leads to severe angiogenic sprouting defects in OIR models ${ }^{32}$, while Robo4 deletion leads to enhanced sprouting angiogenesis (Fig. 2), suggesting that Robo4 could restrain ocular vascularization by blocking Slit2 signalling through Robo1 and 2. However, in contrast to Slit2 mutant mice, neither Robo4 ${ }^{-/-}$nor Robo1 ${ }^{-/-}$; Robo4 $4^{-l-}$ showed any developmental angiogenesis defects ${ }^{32}$. Moreover, our signalling experiments in HUVECs failed to reveal any effect of ROBO4 knockdown or Robo $4 \triangle \mathrm{CD}$ expression on Slit2 signalling. Instead, we find that Slit2 activation of downstream AKT can be completely blocked by ROBO1 and 2 knockdowns or ROBO1 $\triangle \mathrm{CD}$ expression. These data are fully consistent with genetic evidence from studies with Slit2 and Robol and 2 receptor loss-of-function mutants ${ }^{32}$ and reveal opposing actions of Robol, 2 and 4 in ocular vascularization in vivo: Slit2 signals through Robol and 2 to promote OIR angiogenesis, while Robo4 restrains this process independently of its cytoplasmic domain. Thus, heterodimerization of ROBO1 and
4 does not appear to play a biological role in the context of angiogenesis in vivo.

Although ROBO4 failed to show effects on Slit2 signalling, it readily affected VEGF signalling by targeting a specific VEGFR2 intracellular tyrosine, Y951, which controls VEGF-induced c-Src activation, vascular permeability and pathological angiogenesis ${ }^{22-25}$. Since ROBO4 knockdown enhanced VEGFinduced VEGFR2 Y951 and downstream Src phosphorylation, $\mathrm{ROBO} 4$ appears to function as a selective inhibitor of this site's activation. By contrast, ROBO4 fails to affect other intracellular VEGFR2 phosphosites including Y1175, which is critical for PLC- $\gamma$ and downstream ERK activation ${ }^{57}$. Exchange of the Y1173 residue in mouse VEGFR2 (Y1175 in human VEGFR2) for phenylalanine results in arrested endothelial cell development and embryonic death, similar to the global Vegfr2-1phenotype ${ }^{58}$. Selective targeting of Y951 but not Y1175 by ROBO4 is consistent with the phenotype of Robo4 ${ }^{-/-}$mice and may explain why these mice are deficient in angiogenesis under pathological condition, but not angiogenesis during development.

Expression of Robo4 $\triangle C D$ was sufficient to rescue VEGFinduced Y951/Y949 activation and Src phosphorylation in vitro and in vivo, demonstrating that the ROBO4 ICD is dispensable for its effects on VEGF signalling. We had previously shown that the ROBO4 ECD binds to the ECD of another endothelial guidance receptor $\mathrm{UNC} 5 \mathrm{~B}^{18}$, prompting us to investigate if ROBO4 could signal through UNC5B to counteract VEGFR2 Y951 activation. Indeed, we find that like $\mathrm{ROBO}$ knockdown, UNC5B knockdown enhances Y951 but not Y1175 phosphorylation in response to VEGF and that re-expression of Unc5B-FL rescues Y951 activation in UNC5B but not in ROBO4 knockdown cells, supporting a function of UNC5B downstream of ROBO4. Structure-function analysis showed that effects on Y951 depend on the UPA domain in the UNC5B ICD, together supporting a model where ROBO4 affects Y951 by binding and signalling through UNC5B UPA.

How UPA signals remains to be clarified. The domain organization pattern of the UNC5 ICD (that is, ZU5-UPA-DD) is also found in ankyrins, scaffolding proteins regulating the assembly of specialized membrane microdomains ${ }^{59}$, and PIDD, scaffolding proteins controlling programmed cell death ${ }^{60-62}$. Crystal structure analysis has shown that when engaged with ligand (such as endothelial ROBO4), the UNC5B ZU5 and DD domains adopt a closed conformation, leaving the UPA domain exposed and thus able to interact with cytoplasmic signalling molecules ${ }^{51}$. UPA contains several tyrosines that might constitute docking sites for kinases or phosphatases regulating VEGFR2 Y951, but the molecular details remain to be established. Of note, our demonstration that UPA is sufficient to mediate ROBO4-UNCB5 effects on Y951 indicate a critical role for this domain and allow focusing future experiments on this domain.

Figure 7 | ROBO4 regulates VEGF signalling through UNC5B. (a) Proposed model for ROBO4 inhibition of VEGFR2 Y951-Src phosphorylation via the UNC5B UPA domain. (b) Western blot analysis of VEGFR2 pY951 and pY1175 in HUVECs transfected with Ctrl or UNC5B siRNA and treated with 3 nM VEGF. (c) Quantifications of blots shown in b. \#: VEGF significantly induces pY951 and pY1175 at 5 min. UNC5B siRNA further enhances pY951 but not pY1175. $N=5$ experiments, Error bars: s.e.m. ${ }^{\star \star \star} P<0.001$, Student's $t$-test. (d) Schematic diagram showing the engineering of various CD-truncated UNC5B mutants. (e) Western blot analysis and (f) quantifications of pY951 and p-Src in HUVECs with indicated siRNA transfection and adenovirus infection after $3 \mathrm{nM}$ VEGF stimulation. \#: VEGF significantly induces pY951 and p-Src at 5 min. Unc5BFL rescues increased pY951 and p-Src in UNC5B silenced cells. $N=3$ experiments. Error bars: s.e.m. ${ }^{\star} P<0.05{ }^{\star} P<0.01$, Student's $t$-test. (g) Western blot analysis and (h) quantification of $p V E G F R 2$ in HUVECs with indicated siRNA transfection and adenovirus infection after $3 \mathrm{nM}$ VEGF stimulation. \#: VEGF significantly induces pY951 and pY1175 at 5 min. Unc5B $\triangle D D$ and Unc5BUPA but not Unc5B $\triangle C D$ and Unc5BAUPA rescue the excessive VEGFR2 Y951 phosphorylation in UNC5B knockdown cells. None of the constructs affect $\mathrm{Y} 1175$ phosphorylation. $N=3$ experiments. Error bars: s.e.m. ${ }^{\star} P<0.05 ;{ }^{\star \star} P<0.01$, Student's $t$-test. (i) Western blot analysis and (j) quantification of pY951 $(n=3)$ in HUVECs with indicated siRNA transfection and adenovirus infection after 3 nM VEGF stimulation. \#: VEGF significantly induces pY951 at $5 \mathrm{~min}$. Note that expression of Unc5BFL could not rescue the excessive VEGFR2 Y951 phosphorylation in UNC5B and ROBO4 double siRNA-transfected cells. Error bars: s.e.m. ${ }^{\star} P<0.05$, Welch's $t$-test. 
In addition to Robo4, Unc5B binds to various other secreted ligands and transmembrane molecules including Netrins, BMP family members, Neogenin and the fibronectin and leucine-rich transmembrane protein Flrt3 (refs 63-67). Of these, Flrt3 is also expressed in endothelial cells, and conditional Flrt3 knockout mice develop developmental retinal hypervascularization similar to that seen in Unc5B-mutant mice ${ }^{18,68}$. Thus, in contrast to Robo4 mutants, which show normal developmental angiogenesis, both Flrt3 and Unc5B mutants show impaired developmental angiogenesis, suggesting that additional signalling pathways relevant to vascular development are targeted by this interaction. While full elucidation of Unc5B signalling is beyond the scope of the current work, the data shown here demonstrate that endothelial ROBO4 targets VEGFR2 Y951 via the UNC5B UPA domain, thereby counteracting Y951-mediated vessel permeability and angiogenesis. Selectively enhancing VEGFR2 Y951 activation by blocking ROBO4 function targets revascularization without affecting neovascular tuft formation. The reason for this differential behaviour might be because tuft formation involves proliferation, which is not regulated by Y951 but via the VEGFR2 1175 residue. Taken together, we propose that Robo4 blockade might represent an opportunity to accelerate revascularization in retinopathy of prematurity and wound healing in diabetic patients.

\footnotetext{
Methods

Mice. The Yale University Institutional Animal Care and Use Committee (IACUC) reviewed and approved all mouse experiments.

Transgenic mice were generated at Yale Animal Genomics Services Centre. A mouse Robo4 fragment lacking $>95 \%$ of the cytoplasmic domain (aa 1-522) was fused with GFP (Supplementary Fig. 1) and inserted 3' to the tetracyclineresponsive element (TRE)-CMV promoter of the TET-off vector (Clontech). The resulting vector was linearized, purified and microinjected into mouse donor zygotes that were implanted into pseudo-pregnant mice. The resulting founder TET-Robo4 $\Delta$ CD-GFP transgenic mice (C57BL/6J X SJL/J) were propagated on a C57BL/6J background and intercrossed with Cdh5-tTA transgenic mice ${ }^{50}$. Genotyping was performed by detecting the GFP-tag, and the Cdh5-tTA transgene (Supplementary Table 2) $)^{52}$. Robo4 ${ }^{-1-}$ mice on a C57BL/6J background were described previously ${ }^{18}$. To express Robo4 $\Delta \mathrm{CD}$ without endogenous Robo4, Cdh5-tTA;Robo4 $\triangle C D$ transgenic mice were intercrossed with Robo4 ${ }^{-/-}$mice.
}

Antibodies and reagents. The detailed information of all antibodies used in this study is listed in Supplementary Table 3.

Recombinant VEGF-A165 (\#293-VE) and Slit2 (\#5444-SL) were from R\&D Systems (Minneapolis, MN). The AllStars negative control siRNA (\#SI03650318) was from Qiagen (Valencia, CA). Human ROBO1 (SMARTpool: ON-TARGETplus ROBO1 siRNA, \#L-011381-00), ROBO2 (SMARTpool: ONTARGETplus ROBO2 siRNA, \#L-023273-01), ROBO4 (SMARTpool: ON-TARGETplus ROBO4 siRNA, \#L-015216-01) and UNC5B (SMARTpool: ON-TARGETplus UNC5B siRNA, \#L-021392-00) siRNAs (Supplementary Table 1) were from Thermo Scientific (Waltham, MA). Anti-mouse, anti-rabbit and anti-goat secondary Abs conjugated to horseradish peroxidase (HRP) were from Vector Laboratories (Burlingame, CA). Sheep anti-rat IgG conjugated Dynabeads (\#11035), lysine fixable rhodamine dextran (70 kDa MW, \#D1818) and Isolectin B4 (IsoB4) conjugated to Alexa Fluor 488, 546 or 647 were from Invitrogen (Grand Island, NY). Human fibrinogen (\#F8630), heparin (\#H3149), thrombin (\#T9549) and histamine (\#H7125) were from Sigma-Aldrich (St Louis, MO).

Cell culture and cell isolation. Primary MLECs were prepared as previously described $^{69}$. Briefly, mouse lungs were dissected, minced and digested in $1 \mathrm{mg} \mathrm{ml}^{-1}$ type I collagenase (Worthington Biochem. Corp., \#LS004196) for $1 \mathrm{~h}$. Endothelial cells were purified using CD31 Ab-coated Dyna-beads and cultured in DMEM supplemented with $10 \%$ fetal bovine serum (FBS), 100 units $/ \mathrm{ml}$ penicillin, $100 \mu \mathrm{g} \mathrm{ml}^{-1}$ streptomycin, $100 \mu \mathrm{g} \mathrm{ml}^{-1}$ endothelial cell mitogen (Biomedical Technologies, Inc, \#BT-203), and 10 units per ml heparin. After 5-7 days, endothelial cells were further enriched by ICAM-2 Ab-coated Dyna-beads to more than $90 \%$ purity.

HUVECs were obtained from Yale Vascular Biology \& Therapeutics (VBT) program and maintained in medium supplemented with $2 \%$ FBS, VEGF and other necessary growth factors and cytokines (EGM-2 BulletKit, Lonza Inc., Rockland, $\mathrm{ME})$. The cells were verified by CD31 and VE-cadherin staining for endothelial cell identify. Wi-38 fibroblasts were purchased from ATCC and maintained in DMEM containing $10 \%$ FBS, 100 units per ml penicillin, $100 \mu \mathrm{g} \mathrm{ml}^{-1}$ streptomycin, $2 \mathrm{mM}$
L-glutamine and $1 \mathrm{mM}$ sodium pyruvate. All cells were free of mycoplasma contamination.

Adenoviral constructs and recombinant protein. Full-length (FL) and cytoplasmic deleted $(\triangle \mathrm{CD})$ mouse Robo4 constructs were obtained by PCR from mouse Robo4 cDNA (Robo4FL: aa 1-1015; Robo4 $\Delta$ CD: aa 1-522) and fused to mCherry (Supplementary Fig. 1). Rat Robol full length (Robo1FL) (aa 27-1,651) and Robo1 $\triangle C D$ (aa 27-941) constructs were generated by PCR from rat cDNA and fused to IgK signal peptide (METDTLLLWVLLLWVPGSTGD) and GFP (Supplementary Fig. 1). Unc5BFL and truncated Unc5B constructs $(\triangle \mathrm{CD}$ : aa 1-424; $\triangle \mathrm{DD}$ : aa 1-852, $\triangle \mathrm{UPA}$ : aa $1-652$ and UPA: aa $1-538+688-838)$ were obtained by PCR from rat Unc5B cDNA and fused to GFP (Fig. 7e). The fusions were subcloned into pENTR1A vector (Invitrogen) and then transferred into pAd/CMV/V5/DEST using the Gateway Cloning System (Invitrogen, \#V49320). To produce adenoviruses, the constructs were transfected into HEK293 cells and the adenovirus containing supernatants were collected. After titration, the viruses were used to infect cells.

Recombinant mouse sRobo4 (aa 1-478) construct was generated by PCR from mouse Robo4 cDNA and cloned into the eukaryotic expression vector pRK5 as fusion to a C-terminal 6x Histidine tag (sRobo4-His). The construct was transiently transfected into $\mathrm{CHO}$ cells to produce the protein. The recombinant protein was purified to $>95 \%$ purity by affinity chromatography using NiNTA affinity purification (NiNTA Superflow, Qiagen) and reconstituted in PBS/0.2 M NaCl.

ELISA. ELISA was performed using human anti Robo4-1 $\mathrm{mAb}^{18}$ and a biotinylated anti-Robo4 Ab (R\&D Systems, \#BAF2366) as coating and detection $\mathrm{Ab}$, respectively. In brief, 96-well plates (Nunc) were precoated with $1 \mu \mathrm{g} \mathrm{ml}^{-1}$ anti-Robo4-1 mAb overnight, washed with PBST (0.05\% Tween-20 in PBS) and blocked with $\mathrm{PBS} / 2 \%$ goat serum $/ 1 \%$ BSA. Thereafter, each well was incubated with $100 \mu \mathrm{l}$ mouse serum sample for $2 \mathrm{~h}, 100 \mu \mathrm{l}$ of $0.5 \mu \mathrm{g} \mathrm{ml}^{-1}$ biotinylated anti-Robo4 Ab (diluted in PBS/1\% BSA) for $2 \mathrm{~h}$ and streptavidin-HRP (diluted in $\mathrm{PBS} / 1 \% \mathrm{BSA}$ ) for $30 \mathrm{~min}$ at room temperature, with appropriate PBST washes after each incubation. The wells were developed with ELISA development substrate mix (R\&D Systems) for 10-20 min, and the reaction was stopped with stop solution followed by absorbance reading at $450 \mathrm{~nm}$. Concentrations of sRobo4 in mouse serum were calculated using a standard curve generated from mouse sRobo4-HIS.

Immunoprecipitation. Cell culture supernatants, and human or mouse serum were precleared twice with protein A/G magnetic beads (Thermo Scientific, \#88803) and incubated with anti-Robo4-1 mAb in the presence of appropriate protease inhibitors overnight at $4{ }^{\circ} \mathrm{C}$, followed by $1 \mathrm{~h}$ incubation with protein A/G beads. Precipitates were washed three times in PBS, resolved in 2XLaemmli's sample buffer and sRobo4 was detected by Western blot using $\mathrm{R} \& \mathrm{D}$ anti-Robo4 Ab

Whole-mount staining of retinas. The eyes of mouse pups were fixed in $4 \%$ PFA for $20 \mathrm{~min}$. The retinas were dissected and incubated with fluorescent labelled-IsoB4 in Pblec buffer ( $1 \mathrm{mM} \mathrm{MgCl}_{2}, 1 \mathrm{mM} \mathrm{CaCl}_{2}, 0.1 \mathrm{mM} \mathrm{MnCl}, 1 \%$ Triton X-100 in PBS) overnight. After $3 \times 15$ min washing with PBS, the retinas were mounted in fluorescent mounting medium (DAKO Inc.).

Oxygen-induced retinopathy. OIR was performed as described ${ }^{20,21}$. Briefly, the breeding mother and P7 neonatal pups of both genders were exposed to $75 \% \mathrm{O}_{2}$ until P12. The pups were then exposed to room air for an additional 5 days until P17. In some experiments, P12 OIR pups were subjected to intraperitoneal (I.P.) injection of $300 \mathrm{\mu g} \mathrm{g}^{-1}$ recombinant mouse sRobo4 from P12 to P16. Eyes were collected at P17 and the retinas were stained with IsoB4. The avascular, sprouting, tuft and total retina areas were measured using ImageJ.

To test OIR retinal vessel leakage, a mixture of $70 \mathrm{kDa}$ fixable rhodaminedextran (200 $\mathrm{mg} \mathrm{kg}^{-1}$ body weight) and fluorescent Alexa 647-conjugated IsoB4 ( $5 \mathrm{mg} \mathrm{kg}^{-1}$ body weight) was injected into P17 OIR mice (5-6 g) retro-orbitally. Mice were sacrificed after $5 \mathrm{~min}$ or as indicated. The eyes were fixed in $4 \%$ PFA for $20 \mathrm{~min}$. The retinas were dissected and incubated with fluorescent Alexa 488conjugated IsoB4 in Pblec buffer for $10 \mathrm{~h}$. Stained retinas were flat-mounted and imaged with Alexa 488, rhodamine and Alexa 647 channels. To quantify dextran retained in vessels, mean fluorescent intensity of rhodamine in Alexa 647-labelled vessel area was measured using image J, followed by normalization with Alexa 647 intensity.

Miles vascular permeability assay. Six-to-eight week old mice of both genders were injected intravenously with $100 \mu \mathrm{l}$ PBS containing 1\% Evans blue. $15 \mathrm{~min}$ afterwards, $50 \mathrm{ng}$ VEGF (in $20 \mu \mathrm{l}$ saline) or $50 \mathrm{ng}$ histamine (in $20 \mu \mathrm{l} \mathrm{PBS}$ ) was injected intradermally into the shaved back skin. After $30 \mathrm{~min}$, the animals were perfused with $20 \mathrm{ml}$ PBS, and back skin was dissected and imaged. To extract the dye, skin biopsies were collected, dried, weighed and incubated in formamide solution at $56^{\circ} \mathrm{C}$ overnight. The absorbance of the extracts was measured with a spectrophotometer at $620 \mathrm{~nm}$. Evans blue content was calculated using a standard 
curve of Evans blue in formamide and normalized to saline/PBS injected control wild-type mice.

In vivo wound healing assay. Mouse in vivo wound healing assay was described previously ${ }^{70}$. Eight-to-ten week old male mice were shaved and depilated on the back. Wounds were created by a 6 -mm biopsy punch on the back skin. Wound images were acquired with a Leica M125 microscope equipped with a digital camera on days 0 and 7 . Wound areas were measured by ImageJ and presented as percentage of the initial wound area at day 0 .

In vitro sprouting assay. In vitro endothelial cell sprouting assays were performed as described ${ }^{70}$. Briefly, a layer of fibrin was made on the bottom of 24 -well plates by mixing solubilized fibrinogen $\left(10 \mathrm{mg} \mathrm{ml}^{-1}\right.$ in EBM-2) with thrombin (final concentration $1 \mathrm{U}$ ). SiRNA-transfected and adenovirus infected HUVECs were suspended in fibrinogen solution and plated on top of the fibrin gel. After the upper layer of gel was solidified, Wi-38 fibroblasts were plated on the top. Cells were replenished with EBM-2 supplemented with 2\% FBS in the presence of VEGF or Slit2 for 5 days. After trypsinizing fibroblasts on the top, sprouts were labeled with $4 \mathrm{mg} \mathrm{ml}^{-1}$ Calcein (Life Technologies) for $1 \mathrm{~h}$, photographed with a fluorescence microscope and the area covered by endothelial sprouts measured by ImageJ.

In vitro endothelial permeability assay. HUVEC permeability assay was performed in 24-well plates containing transwell inserts $(6.5-\mathrm{mm}$ diameter, $0.4-\mu \mathrm{m}$ pore size polycarbonate filters, Corning Costar Corporation) according to manufacturer's instruction. In brief, HUVECs were transfected with siRNA on day 0 and infected with GFP or Robo4 adenovirus on day 1. On day 2, cells were plated onto fibronectin $\left(10 \mu \mathrm{g} \mathrm{ml}^{-1}\right)$-coated transwell inserts at a density of 150,000 cells per $\mathrm{cm}^{2}$ and cultured in complete media for 3 days to allow formation of cell monolayer. To test transwell permeability, FITC-dextran $(70 \mathrm{kDa}$, Molecular Probes) with final concentration of $1 \mathrm{mg} \mathrm{ml}^{-1}$ and $5 \mathrm{nM}$ VEGF was added into the upper compartment of the inserts. After $30 \mathrm{~min}$. FITC-dextran intensity in the medium of the lower compartment was measured using a fluorescence spectrophotometer.

In vitro wound healing assay. HUVECs were transfected and infected with the indicated siRNA and adenovirus. After $48 \mathrm{~h}$, cells were plated into 6-well plates, cultured until confluence and starved overnight in $0.2 \% \mathrm{FBS} / \mathrm{EBM}-2$. Scratch wounds were created with a $200-\mu \mathrm{l}$ pipette tip. The wounded cell monolayer was cultured in $0.2 \%$ FBS/EBM-2 supplemented with $6 \mathrm{nM}$ VEGF-A. Cell-free areas were photographed at $0 \mathrm{~h}$ and $20 \mathrm{~h}$ post wounding under an inverted light microscope connected with a digital camera.

Quantitative real-time PCR. Total RNAs were purified from HUVECs using the RNeasy Plus Mini Kit (Qiagen, \#74134) and reverse transcribed to cDNAs using IScript Reverse Transcriptase III (Bio-Rad, \#170-8891) according to manufacturers' instructions. QRT-PCR was performed using the resulting cDNAs and the corresponding primers (Supplementary Table 2). The data were first normalized to GAPDH, and the relative expression levels of different genes were calculated.

Signalling studies. HUVECs were transfected with 20 pmol siRNA per well in 6-well plates using RNAiMax transfection reagent (Invitrogen, \#13778) according to the manufacturer's instruction. Infection with GFP, Robo4 or Unc5B adenovirus was done one day after siRNA transfection. After $48 \mathrm{~h}$, cells were starved $10 \mathrm{~h}$ to overnight in EBM-2 supplemented with $0.2 \%$ FBS and treated with $1 \mu \mathrm{g} \mathrm{ml}^{-1}$ $(6 \mathrm{nM})$ recombinant Slit2 or $25 \mathrm{ng} \mathrm{ml}^{-1}(3 \mathrm{nM})$ VEGF-A. For MLEC signalling experiments, primary MLECs were starved $10 \mathrm{~h}$ to overnight in DMEM containing $2 \%$ FBS and stimulated with $5 \mathrm{nM}$ VEGF-A. Cell lysates were collected at the indicated time points and subjected to $4-15 \%$ SDS-PAGE followed by immunoblotting using appropriate primary and second Abs. See Supplementary Fig. 8 for all uncropped immunoblots.

Statistics. No statistical methods were used to determine sample size before experiments. Animals were selected for animal experiments based on their genotypes and proper age and gender as described in 'Methods' section, which was a pre-established criterion before the experiment. No randomization and blinding were used. Samples with equal variances were tested using Mann-Whitney $U$ test or two-tailed Student's $t$-test between groups. When the variances were unequal, samples were tested using Welch's $t$-test. $P$ value $<0.05$ was considered to be statistically significant.

Data availability. All data that support the findings of this study are available within the article and its Supplementary Information File and from the corresponding author upon reasonable request.

\section{References}

1. Huminiecki, L., Gorn, M., Suchting, S., Poulsom, R. \& Bicknell, R. Magic roundabout is a new member of the roundabout receptor family that is endothelial specific and expressed at sites of active angiogenesis. Genomics 79, 547-552 (2002).

2. Park, K. W. et al. Robo4 is a vascular-specific receptor that inhibits endothelial migration. Dev. Biol. 261, 251-267 (2003).

3. Cai, H. et al. Roundabout 4 regulates blood-tumor barrier permeability through the modulation of ZO-1, Occludin, and Claudin-5 expression. J. Neuropathol. Exp. Neurol. 74, 25-37 (2015).

4. Nafar, M. et al. The novel diagnostic biomarkers for focal segmental glomerulosclerosis. Int. J. Nephrol. 2014, 574261 (2014).

5. Okada, Y. et al. A three-kilobase fragment of the human Robo4 promoter directs cell type-specific expression in endothelium. Circ. Res. 100, 1712-1722 (2007).

6. Zhuang, X., Cross, D., Heath, V. L. \& Bicknell, R. Shear stress, tip cells and regulators of endothelial migration. Biochem. Soc. Trans. 39, 1571-1575 (2011).

7. Okada, Y. et al. A GABP-binding element in the Robo4 promoter is necessary for endothelial expression in vivo. Blood 112, 2336-2339 (2008).

8. Okada, Y. et al. Expression of the Robo4 receptor in endothelial cells is regulated by two AP-1 protein complexes. Biochem. Biophys. Res. Commun. 467, 987-991 (2015).

9. Yoshikawa, M. et al. Robo4 is an effective tumor endothelial marker for antibody-drug conjugates based on the rapid isolation of the anti-Robo4 cell-internalizing antibody. Blood 121, 2804-2813 (2013).

10. Goto-Koshino, Y. et al. Robo4 plays a role in bone marrow homing and mobilization, but is not essential in the long-term repopulating capacity of hematopoietic stem cells. PLoS ONE 7, e50849 (2012).

11. Smith-Berdan, S. et al. Robo4 cooperates with CXCR4 to specify hematopoietic stem cell localization to bone marrow niches. Cell Stem Cell 8, 72-83 (2011).

12. Smith-Berdan, S., Schepers, K., Ly, A., Passegue, E. \& Forsberg, E. C. Dynamic expression of the Robo ligand Slit2 in bone marrow cell populations. Cell Cycle 11, 675-682 (2012).

13. Smith-Berdan, S., Nguyen, A., Hong, M. A. \& Forsberg, E. C. ROBO4-mediated vascular integrity regulates the directionality of hematopoietic stem cell trafficking. Stem Cell Rep. 4, 255-268 (2015).

14. Suchting, S., Heal, P., Tahtis, K., Stewart, L. M. \& Bicknell, R. Soluble Robo4 receptor inhibits in vivo angiogenesis and endothelial cell migration. FASEB J. 19, 121-123 (2005).

15. Yadav, S. S. \& Narayan, G. Role of ROBO4 signalling in developmental and pathological angiogenesis. Biomed. Res. Int. 2014, 683025 (2014).

16. Zhuang, X. et al. Robo4 vaccines induce antibodies that retard tumor growth. Angiogenesis 18, 83-95 (2015).

17. Jones, C. A. et al. Robo4 stabilizes the vascular network by inhibiting pathologic angiogenesis and endothelial hyperpermeability. Nat. Med. 14, 448-453 (2008)

18. Koch, A. W. et al. Robo4 maintains vessel integrity and inhibits angiogenesis by interacting with UNC5B. Dev. Cell 20, 33-46 (2011).

19. Marlow, R. et al. Vascular Robo4 restricts proangiogenic VEGF signaling in breast. Proc. Natl Acad. Sci. USA 107, 10520-10525 (2010).

20. Smith, L. E. et al. Oxygen-induced retinopathy in the mouse. Invest. Ophthalmol. Vis. Sci. 35, 101-111 (1994).

21. Connor, K. M. et al. Quantification of oxygen-induced retinopathy in the mouse: a model of vessel loss, vessel regrowth and pathological angiogenesis. Nat. Protoc. 4, 1565-1573 (2009).

22. Ruan, G. X. \& Kazlauskas, A. VEGF-A engages at least three tyrosine kinases to activate PI3K/Akt. Cell Cycle 11, 2047-2048 (2012).

23. Matsumoto, T. et al. VEGF receptor-2 Y951 signaling and a role for the adapter molecule TSAd in tumor angiogenesis. EMBO J. 24, 2342-2353 (2005).

24. Sun, Z. et al. VEGFR2 induces c-Src signaling and vascular permeability in vivo via the adaptor protein TSAd. J. Exp. Med. 209, 1363-1377 (2012).

25. Li, X. et al. VEGFR2 pY949 signalling regulates adherens junction integrity and metastatic spread. Nat. Commun. 7, 11017 (2016).

26. Jones, C. A. et al. Slit2-Robo4 signalling promotes vascular stability by blocking Arf6 activity. Nat. Cell Biol. 11, 1325-1331 (2009).

27. Brose, K. et al. Slit proteins bind Robo receptors and have an evolutionarily conserved role in repulsive axon guidance. Cell 96, 795-806 (1999).

28. Kidd, T. et al. Roundabout controls axon crossing of the CNS midline and defines a novel subfamily of evolutionarily conserved guidance receptors. Cell 92, 205-215 (1998).

29. Kidd, T., Bland, K. S. \& Goodman, C. S. Slit is the midline repellent for the robo receptor in Drosophila. Cell 96, 785-794 (1999).

30. Paul, J. D. et al. SLIT3-ROBO4 activation promotes vascular network formation in human engineered tissue and angiogenesis in vivo. J. Mol. Cell. Cardiol. 64, 124-131 (2013).

31. Yuen, D. A. \& Robinson, L. A. Slit2-Robo signaling: a novel regulator of vascular injury. Curr. Opin. Nephrol. Hypertens. 22, 445-451 (2013).

32. Rama, N. et al. Slit2 signaling through Robo1 and Robo2 is required for retinal neovascularization. Nat. Med. 21, 483-491 (2015). 
33. Mommersteeg, M. T. et al. Slit-roundabout signaling regulates the development of the cardiac systemic venous return and pericardium. Circ. Res. 112, 465-475 (2013).

34. Kaur, S. et al. Silencing of directional migration in roundabout 4 knockdown endothelial cells. BMC Cell Biol. 9, 61 (2008).

35. Sheldon, H. et al. Active involvement of Robo1 and Robo4 in filopodia formation and endothelial cell motility mediated via WASP and other actin nucleation-promoting factors. FASEB J. 23, 513-522 (2009).

36. Morlot, C. et al. Structural insights into the Slit-Robo complex. Proc. Natl Acad. Sci. USA 104, 14923-14928 (2007).

37. Fukuhara, N., Howitt, J. A., Hussain, S. A. \& Hohenester, E. Structural and functional analysis of slit and heparin binding to immunoglobulin-like domains 1 and 2 of Drosophila Robo. J. Biol. Chem. 283, 16226-16234 (2008).

38. Hussain, S. A. et al. A molecular mechanism for the heparan sulfate dependence of slit-robo signaling. J. Biol. Chem. 281, 39693-39698 (2006).

39. Zelina, P. et al. Signaling switch of the axon guidance receptor Robo3 during vertebrate evolution. Neuron 84, 1258-1272 (2014).

40. Wang, B. et al. Induction of tumor angiogenesis by Slit-Robo signaling and inhibition of cancer growth by blocking Robo activity. Cancer Cell 4, 19-29 (2003).

41. Zhang, B. et al. Repulsive axon guidance molecule Slit3 is a novel angiogenic factor. Blood 114, 4300-4309 (2009).

42. Urbich, C. et al. HDAC5 is a repressor of angiogenesis and determines the angiogenic gene expression pattern of endothelial cells. Blood 113, 5669-5679 (2009).

43. Dunaway, C. M. et al. Cooperative signaling between Slit2 and Ephrin-A1 regulates a balance between angiogenesis and angiostasis. Mol. Cell. Biol. 31, 404-416 (2011).

44. Fish, J. E. et al. A Slit/miR-218/Robo regulatory loop is required during heart tube formation in zebrafish. Development 138, 1409-1419 (2011).

45. Small, E. M., Sutherland, L. B., Rajagopalan, K. N., Wang, S. \& Olson, E. N. MicroRNA-218 regulates vascular patterning by modulation of Slit-Robo signaling. Circ. Res. 107, 1336-1344 (2010).

46. Larrivee, B. et al. Activation of the UNC5B receptor by Netrin-1 inhibits sprouting angiogenesis. Genes Dev. 21, 2433-2447 (2007).

47. Bouvree, K. et al. Netrin-1 inhibits sprouting angiogenesis in developing avian embryos. Dev. Biol. 318, 172-183 (2008).

48. Castets, M. et al. Inhibition of endothelial cell apoptosis by netrin-1 during angiogenesis. Dev. Cell 16, 614-620 (2009).

49. Lu, X. et al. The netrin receptor UNC5B mediates guidance events controlling morphogenesis of the vascular system. Nature 432, 179-186 (2004).

50. Sun, J. F. et al. Microvascular patterning is controlled by fine-tuning the Akt signal. Proc. Natl Acad. Sci. USA 102, 128-133 (2005).

51. Wang, R. et al. Autoinhibition of UNC5b revealed by the cytoplasmic domain structure of the receptor. Mol. Cell 33, 692-703 (2009).

52. Donaldson, J. G. Multiple roles for Arf6: sorting, structuring, and signaling at the plasma membrane. J. Biol. Chem. 278, 41573-41576 (2003).

53. Vehlow, A. et al. Endophilin, Lamellipodin, and Mena cooperate to regulate F-actin-dependent EGF-receptor endocytosis. EMBO J. 32, 2722-2734 (2013).

54. Galovic, M., Xu, D., Areces, L. B., van der Kammen, R. \& Innocenti, M. Interplay between N-WASP and CK2 optimizes clathrin-mediated endocytosis of EGFR. J. Cell Sci. 124, 2001-2012 (2011).

55. Gryaznova, T. et al. Intersectin adaptor proteins are associated with actin-regulating protein WIP in invadopodia. Cell Signal. 27, 1499-1508 (2015).

56. Burke-Gaffney, A., Svermova, T., Mumby, S., Finney, S. J. \& Evans, T. W. Raised plasma Robo4 and cardiac surgery-associated acute kidney injury. PLoS ONE 9, e111459 (2014).

57. Koch, S. \& Claesson-Welsh, L. Signal transduction by vascular endothelial growth factor receptors. Cold Spring Harb. Perspect Med. 2, a006502 (2012).

58. Sakurai, Y., Ohgimoto, K., Kataoka, Y., Yoshida, N. \& Shibuya, M. Essential role of Flk-1 (VEGF receptor 2) tyrosine residue 1173 in vasculogenesis in mice. Proc. Natl Acad. Sci. USA 102, 1076-1081 (2005).

59. Bennett, V. \& Healy, J. Organizing the fluid membrane bilayer: diseases linked to spectrin and ankyrin. Trends Mol. Med. 14, 28-36 (2008).
60. Cuenin, S., Tinel, A., Janssens, S. \& Tschopp, J. p53-induced protein with a death domain (PIDD) isoforms differentially activate nuclear factor-kappaB and caspase-2 in response to genotoxic stress. Oncogene 27, 387-396 (2008).

61. Lin, Y., Ma, W. \& Benchimol, S. Pidd, a new death-domain-containing protein is induced by p53 and promotes apoptosis. Nat. Genet. 26, 122-127 (2000).

62. Park, H. H. et al. Death domain assembly mechanism revealed by crystal structure of the oligomeric PIDDosome core complex. Cell 128, 533-546 (2007).

63. Leonardo, E. D. et al. Vertebrate homologues of C. elegans UNC-5 are candidate netrin receptors. Nature 386, 833-838 (1997).

64. MacNeil, L. T., Hardy, W. R., Pawson, T., Wrana, J. L. \& Culotti, J. G. UNC-129 regulates the balance between UNC-40 dependent and independent UNC-5 signaling pathways. Nat. Neurosci. 12, 150-155 (2009).

65. Lejmi, E. et al. Netrin-4 inhibits angiogenesis via binding to neogenin and recruitment of Unc5B. Proc. Natl Acad. Sci. USA 105, 12491-12496 (2008).

66. Hagihara, M. et al. Neogenin, a receptor for bone morphogenetic proteins. J. Biol. Chem. 286, 5157-5165 (2011).

67. Yamagishi, S. et al. FLRT2 and FLRT3 act as repulsive guidance cues for Unc5-positive neurons. EMBO J. 30, 2920-2933 (2011).

68. Seiradake, E. et al. FLRT structure: balancing repulsion and cell adhesion in cortical and vascular development. Neuron 84, 370-385 (2014).

69. Zhang, F. et al. Tetraspanin CD151 maintains vascular stability by balancing the forces of cell adhesion and cytoskeletal tension. Blood 118, 4274-4284 (2011).

70. Dubrac, A. et al. Targeting NCK-mediated endothelial cell front-rear polarity inhibits neovascularization. Circulation 133, 409-421 (2016).

\section{Acknowledgements}

This project was supported by grants from NHLBI (1R01HLI125811), NEI (1R01EY025979-01) and the Agence Nationale de la Recherche (ANR-GANDHI, ANR-NOVA). Feng Zhang was supported by a T32 grant from NHLBL (5T32HL007950-15). We thank Federico Corti, Jinah Han, Roxana Ola, June-Hee Park and Georgia Zarkada for technical support and discussion.

\section{Author contributions}

F.Z., C.P., T.M., L.P.-F., J.Z., G.G. and A.D. performed experiments and analysed data. A.E., F.Z. and C.P. designed experiments, analysed data and wrote the manuscript.

\section{Additional information}

Supplementary Information accompanies this paper at http://www.nature.com/ naturecommunications

Competing financial interests: The authors declare no competing financial interests.

Reprints and permission information is available online at http://npg.nature.com/ reprintsandpermissions/

How to cite this article: Zhang, F. et al. The Robo4 cytoplasmic domain is dispensable for vascular permeability and neovascularization. Nat. Commun. 7, 13517 doi: 10.1038/ncomms13517 (2016).

Publisher's note: Springer Nature remains neutral with regard to jurisdictional claims in published maps and institutional affiliations.

(c) This work is licensed under a Creative Commons Attribution 4.0 article are included in the article's Creative Commons license, unless indicated otherwise in the credit line; if the material is not included under the Creative Commons license, users will need to obtain permission from the license holder to reproduce the material. To view a copy of this license, visit http://creativecommons.org/licenses/by/4.0/

(C) The Author(s) 2016 